\title{
Localization and text sequence restoration using noise pixels in binary document image watermarking
}

\author{
Niladri B. Puhan \\ Nanyang Technological University \\ School of Computer Engineering \\ Singapore \\ E-mail: puhan@ntu.edu.sg \\ Anthony T. S. Ho \\ University of Surrey \\ Department of Computing \\ Faculty of Engineering and Physical Sciences \\ Guildford Surrey, United Kingdom \\ Farook Sattar \\ Nanyang Technological University \\ School of Electrical and Electronic Engineering \\ Singapore
}

\begin{abstract}
We propose a new method for tamper localization and restoration using noise pixels in binary document images. For such images, it is difficult to find a sufficient number of low-distortion pixels in individual blocks with blind detection property. Also, a perceptual watermark cannot be embedded in white regions of the document image, making such regions insecure against hostile attacks. An erasable watermark is embedded in each block of the document image independently. The embedding process introduces some background noise. However, the content in the document can be interpreted by the user, because human vision has the inherent capability to recognize various patterns in the presence of noise. If authenticity is verified for the content of each block, the exact copy of original image is restored at the blind detector for further use and analysis. Experimental results show that an erasable watermark of necessary data length can be embedded in individual blocks to attain effective localization and restoration capability. Using the proposed method, it is possible to restore the original text sequence in text document images after multiple alterations like text deletion, insertion, substitution, and block swapping. (c) 2009 SPIE and IS\&T. [DOI: 10.1117/1.3143184]
\end{abstract}

\section{Introduction}

In recent times, the information technology revolution has significantly transformed many parts of our lives. With increasing application of digital data in various forms such as image, video, music, documents, and graphics, its security and authentic usage has posed important technological challenges. Intelligent hiding of the watermark within a digital image could make it possible to verify whether it has been misused by malicious attackers after the watermarking

Paper 08063RR received Apr. 17, 2008; revised manuscript received Apr. 11, 2009; accepted for publication Apr. 13, 2009; published online May 21, 2009.

1017-9909/2009/18(2)/023012/21/\$25.00 ๔ 2009 SPIE and IS\&T. process. After extracting the hidden watermark from the image in question, important objectives such as ownership verification, traitor tracing, tamper localization, and restoration can be achieved.

A majority of existing image watermarking methods are for grayscale or color images in which the pixels take on a wide range of values. For those images, changing pixel values by a reasonable margin is imperceptible to the human eye. For images in which the pixels take on only a limited number of values of either 0 (black) or 1 (white), embedding the watermark without any visual distortion becomes more challenging. Binary document images could potentially include digitized versions of text, circuit diagrams, signature, driver licenses, financial and legal documents, maps, and drawings. By using different image processing software, the editing of document images become easier. As such, the ownership protection, authentication, and annotation of binary document images have become important in recent years.

Low et $a .^{2-4}$ introduced robust watermarking methods for formatted document images based on imperceptible line and word shifting. The methods were applied to embed information in document images for bulk electronic publications. The line shifting method was found to have low capacity, but the embedded data was robust to photocopying, scanning, and printing processes. The word shifting method could offer higher capacity than the line shifting method, but the robustness was reduced to printing, photocopying, and scanning. Brassil and O'Gorman proposed a method in Ref. 5 where the height of the bounding box enclosing a group of words could be used as a feature for 
embedding. This method has a better data hiding capacity than the line and word shifting methods. It was also robust to distortions caused by photocopying.

$\mathrm{Wu}$ and Liu hide data in a binary image using a hierarchical model in which human perception was taken into consideration. ${ }^{6}$ Distortion that occurred due to flipping of a pixel was measured by considering the change in smoothness and connectivity of a $3 \times 3$ window centered at the pixel. In a block, the total number of black pixels is modified to be either odd or even for embedding the data bits. Shuffling was used to equalize the uneven embedding capacity over the image. Koch and $\mathrm{Zhao}^{7}$ proposed a data hiding algorithm in which a data bit 1 is embedded if the percentage of white pixels was greater than a given threshold and a data bit 0 is embedded if the percentage of white pixels was less than another given threshold. This algorithm was not robust to attacks, and the hiding capacity was low. Mei et al. modified an eight-connected boundary of a connected component for data hiding. ${ }^{8}$ A fixed set of pairs of 5-pixel-long boundary patterns have been identified for embedding data. A unique property of the method is that the two patterns in each pair are duplicates of each other. This property allowed for blind detection of the watermark in a document image.

Amamo and Misaki proposed a feature calibration method in which text areas in an image were identified and the geometry of the bounding box of each text line was calculated in Ref. 9 Each bounding box was divided into four partitions and grouped into two sets. The average width of the horizontal strokes of characters was modified as a feature. In Ref. 10, a new perceptual measure based on curvature-weighted distance difference (CWDD) measure was proposed toward digital watermarking of binary document images. Puhan and $\mathrm{Ho}^{11}$ proposed a new exact authentication algorithm using the reversible property of the perceptual measure so that the possibility of any undetected content modification is removed. The method embeds an authentication signature computed from the original image into itself after identifying an ordered set of low-distortion pixels. If the flipping of a pixel is imperceptible to the human eye, it is termed as a low-distortion pixel. Such pixels are found in a binary document image by using a suitable perceptual model. The parity attack found in the block-wise data hiding method ${ }^{6}$ becomes infeasible due to pixel-wise embedding of the authentication signature.

Watermarking methods ${ }^{12,13}$ have been suggested for halftone images that are used in printed media such as magazines, newspapers, and printer outputs. These methods are particularly useful for halftone images and are not suitable for other category of document images like text, drawings, and cartoons, which have sharply contrasted boundaries. Robustness to printing, scanning, and photocopying is an important issue when the documents are to be distributed in analog form. The line and word shifting approaches are found to be robust to printing, scanning, and photocopying operations. However, these methods have low capacity, which does not make them suitable for authentication application. The methods using pixel flipping and feature modification approach are not robust to printing and scanning, but they offer higher capacity. These methods are useful in applications when documents are distributed in the electronic form and robustness to distortions is not an issue. An interesting discussion of various watermarking and data hiding methods for binary document images is given in Ref. 14. Some fragile and semifragile watermarking methods were proposed in Refs. 15-17

Most of the binary image watermarking methods deal with the issue of robustness and annotation applications. However, the advantages of authentication watermarking for tamper localization and restoration have yet to be fully achieved. A method for localization in binary document images has been reported by Kim and Queiroz. ${ }^{18}$ In this alteration locating method, the original image was subdivided into many subimages and each subimage was watermarked independently. A two-layer watermark was embedded imperceptibly using a block-wise data hiding technique to verify the integrity of the watermarked image and localizing any modification in it. The size of each subimage was $128 \times 128$ pixels, so its localization accuracy was found to be low. The block-wise embedding technique used in this method suffers from the parity attack. ${ }^{6}$ The parity attack arises because the signature is embedded by considering the parity of the blocks, the number of black pixels. If two pixels that belong to the same block change their values, the parity of this block may not change, and so this modification will pass undetected.

A new localization method has been proposed using a connectivity-preserving transition criterion. ${ }^{19}$ The image is partitioned into multiple macro-blocks, and an adaptive block identifier is embedded in selected macro-blocks for tamper localization. In this method, the localization accuracy is improved to $33 \times 33$ pixels block size. However, some possibility of false tamper detection and incorrect localization exists after attacks.

Previous work on restoration of text document images has been reported by Makur in Ref. 20 In text document images, several characters from a finite set convey the necessary information. Each character of the English alphanumeric set can be represented by a 7-bit American Standard Code for Information Interchange (ASCII) code. In this method, a self-embedding technique was used for restoration of the original character sequence. The ASCII code of a character was used as its watermark and embedded imperceptibly in another character of the document. For watermark embedding, a particular character was selected through a random permutation or cyclic shift function. During watermark verification, each character was compared with its corresponding watermark to restore the original character sequence after tamper localization. This method is effective for restoration against the individual alterations like character substitution, deletion, and insertion. However, false tamper detection, and restoration failure may occur after only a few individual alterations. For multiple alterations such as combined deletion and insertion of characters in the document, restoration is difficult due to a loss of synchronization.

To overcome these shortcomings in tamper localization and restoration in binary document images, a new authentication watermarking method is proposed by constructing an erasable watermark from noise pixels. The proposed method is feasible and effective for localization in binary document images in electronic form. Then the localization method is extended for restoration of the original text sequence in text document images. 
In this paper, we propose new watermarking methods for localization and text sequence restoration in binary document images. The rest of the paper is organized as follows: In Sec. 2, the proposed localization method for binary document images is described. In Sec. 3, the proposed restoration method for text document images is presented. Last, conclusions are drawn in Sec. 4.

\section{Proposed Localization Method}

\subsection{Motivation for Using Erasable Watermark}

In this section, we discuss the motivation behind using an erasable watermark for localization and restoration. For hiding the authentication signature in each block imperceptibly, two requirements should be fulfilled. First, the number of low-distortion pixels in a block to embed the authentication signature should be high. Second, the watermark detection process should be blind. Within a reasonable block size, there is insufficient number of low-distortion pixels available for embedding. The blind detection requirement of these pixels adds to the difficulty in achieving high watermark capacity in each block. Furthermore, an imperceptible watermark cannot be embedded in white regions of the image. The inability to watermark in the white regions makes the detector vulnerable to malicious tampering. Due to these shortcomings, it is evident that unless the block size is large, imperceptible watermarking may not be suitable for block-wise tamper localization.

We pay attention to the possibility of embedding the signature in other such pixels that causes visual distortion in the watermarked image. However, the resulting distortion due to the embedding process can be erased entirely at the blind detector. After erasing the embedded watermark, the original image can be restored at the blind detector. This particular concept is known as erasable, invertible, or reversible watermarking in the literature, and the watermark thus embedded is termed as an erasable watermark. The erasable watermarking algorithm proposed by Fridrich et $a .^{21}$ for image authentication is of particular interest to this paper. The proposed algorithm can be summarized as follows: Let $A$ represent the information that is altered in the cover image when we embed a message of $N$ bits. Fridrich et $a_{l}{ }^{21}$ have shown that the erasability is possible provided $A$ is compressible. If $A$ can be losslessly compressed to $M$ bits, $N-M$ additional bits can be erasably embedded in the cover image. In the implementation of this algorithm for natural images, it is observed that the neighboring pixels are highly correlated. Thus some bit planes in the whole image can be sufficiently compressed to implement an erasable watermark.

For binary document images, each pixel is represented by one bit, and, it can be considered that there is only one bit plane in the image. If all pixels in the bit plane are losslessly compressed to construct the erasable watermark, then the compressed block does not have perceptual correlation with the original. Therefore, creating an erasable watermark by directly compressing the bit plane is not relevant in document images. If a set of suitable pixels with high correlation can be found in a block, they can be losslessly compressed, and an erasable watermark of high capacity can be constructed for document images.

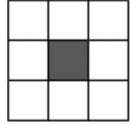

(a)

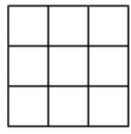

(d)

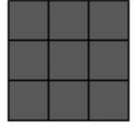

(b)

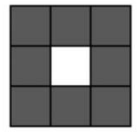

(e)

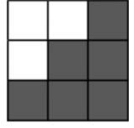

(c)

(f)

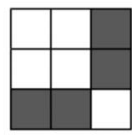

Fig. 1 Different categories of pixels in a binary document image based on their 8-neighborhood.

\subsection{Pixel Selection for Watermark Embedding}

To construct an erasable watermark, we find a set of pixels in each block of the binary image such that (1) there exists a high correlation among the pixels, (2) the same set of pixels can be found at a blind detector, and (3) the relevant information is preserved after the embedding process so that the user can determine whether the particular watermarked image is useful. We analyze pixels in the binary document image based on their 8-neighborhood. As shown in Fig. 1, there can be six categories of pixel neighborhoods in such images. The center pixel can be either black or white with its eight neighbor pixels possibly all black, or all white, or a mixture of black and white. Pixels whose neighborhoods have both white and black pixels are contour pixels like in Figs. 1(c) and 1(f), and they convey important visual information in the document image. The center pixel in Fig. 1(b) is called a foreground pixel, and the center white pixel in Fig. 1(e) can represent a hole, so these pixels convey some information. The black pixel whose all neighbor pixels are white is termed an isolated pixel, as in Fig. 1(a). These pixels are perceived as noise in a binary image. As shown in Fig. 1(d), a white pixel whose all neighbor pixels are white is termed a background pixel.

Among these pixel categories, we choose the isolated and background pixels for embedding an erasable watermark due to following reasons: Isolated and background pixels do not convey important information within document images. If these pixels are altered, a background noise will be formed in the image that is similar to the salt-andpepper noise found in natural images. In document images, we obtain information by recognizing various patterns such as characters, symbols, lines, and curves. It is known that human vision has the remarkable ability to recognize such patterns even in the presence of noise. So after embedding an erasable watermark in these pixels, the user can still obtain relevant information about the document. The background pixels occur in long sequences, and isolated pixels occur between them with less probability. Such a set of pixels can be significantly compressed using the run-length coding scheme. ${ }^{22}$ Flipping of a background pixel creates an isolated pixel and vice versa, so blind detection of the embedded pixels is possible.

To construct the erasable watermark in each block, an ordered set of insignificant pixels are searched in a sequential scanning order starting from left to right and from top to bottom. A pixel within a block is defined as an insignificant pixel if it satisfies the following conditions 1,2 , and 3 . 


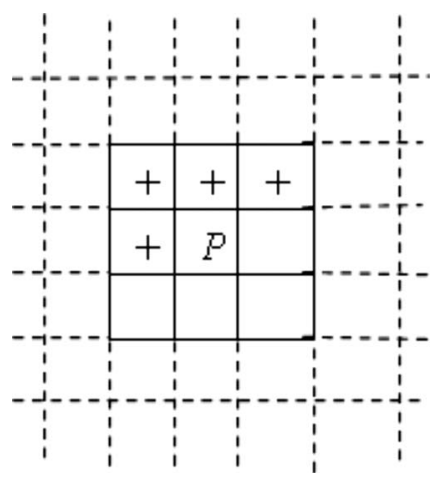

Fig. 2 Pixel grid explaining status change possibility of the pseudoinsignificant pixel $(P)$.

A pixel is defined as a pseudo-insignificant pixel if it satisfies conditions 1 and 2 but does not satisfy condition 3 . The rest of the pixels within a block are defined as residual pixels. The conditions are designed to ensure that after flipping the current pixel, an insignificant pixel should not be detected as a pixel that is not insignificant and vice versa during blind detection. A pixel in a block is defined to be an insignificant pixel if:

Condition 1 The pixel is either a background pixel or an isolated pixel.

Condition 2 In an $M \times M$ pixel window, there should not be any insignificant or pseudo-insignificant pixel already found in the block.

Condition 3 After flipping the current pixel, there should not be any pixel in its 8-pixel neighborhood that comes before in the scanning order and satisfies the preceding two conditions.

Condition 3 is verified as follows. Suppose that we are deciding the status of the pixel $(P)$ during embedding using conditions 1,2 , and 3 in Fig. 2 . If it satisfies condition 1 and 2 , then condition 3 is tested as follows. The pixel $(P)$ is flipped, and then a total of four $(+)$ marked pixels are tested for condition 1 and 2 in scanning order. This is possible since the status of pixels coming before the $(+)$ marked pixels in scanning order is already known. If any $(+)$ marked pixel is found to satisfy conditions 1 and 2 , the rest of such pixels are not tested. Pixel $(P)$ is then identified as a pseudo-insignificant pixel.

\subsubsection{Analysis}

The reason for selecting the background and isolated pixels for constructing the erasable watermark has already been explained in this section. Condition 2 is necessary due to the following reasons: Flipping of the current pixel may cause a change in the status of the already found insignificant pixel, and the pseudo-insignificant pixel already found in its neighborhood may become an insignificant pixel after the flipping process. Thus, a change in the status of already found insignificant and pseudo-insignificant pixels can lead to wrong blind detection; the flipping of the current pixel could change the status of its 8-neighborhood insignificant pixel.

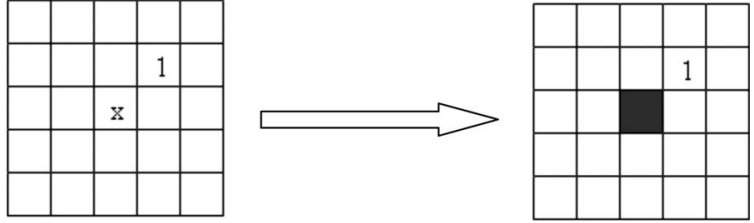

(a)
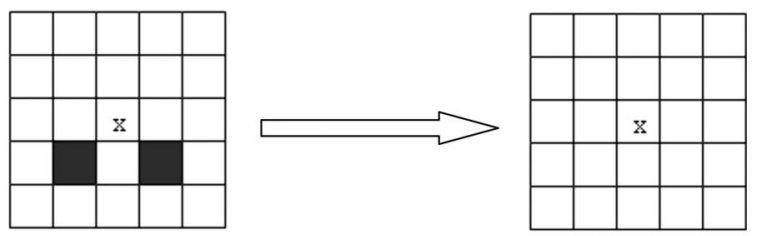

(b)
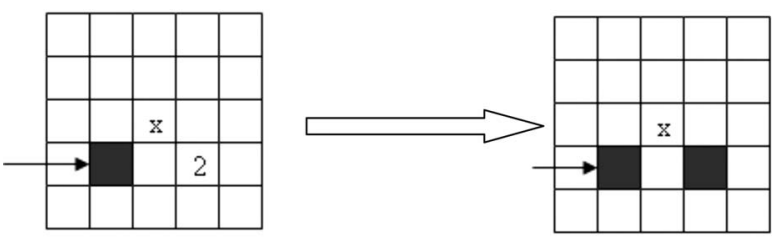

(c)

Fig. 3 Examples of pixel patterns to illustrate the wrong blind detection of insignificant pixels. Left: Patterns before flipping process. Right: Patterns after flipping process.

This possibility is shown in Fig. 3(a) as an example. Flipping the center pixel (marked $x$ ) to black will affect the neighboring pixel (marked 1 ). This pixel no longer satisfies condition 1 after flipping the center pixel. If it has been identified as an insignificant pixel for embedding, its status will be changed during blind detection. In a $3 \times 3$ pixel window centered on a pixel, there should not be more than one insignificant pixel. This is because flipping of multiple insignificant pixels may convert a pixel (that is not insignificant) to an insignificant one. This is shown as an example in Fig. 3(b). If both black insignificant pixels are flipped, then there is a possibility that the center pixel (marked $x$ ) will be detected as an (false) insignificant pixel. In Fig. 3(c), an example is shown where flipping of a white pixel could convert a pseudo-insignificant pixel to an insignificant pixel. When $M$ is equal to 3 , the black pixel (marked with an arrow) satisfies conditions 1 and 2. However, it does not satisfy condition 3 , because the pixel in its 8-neighborhood (marked $x$ ) satisfies conditions 1 and 2 after its flipping. This black pixel is therefore detected as a pseudo-insignificant pixel, and it is not considered for embedding. The white pixel (marked 2) is detected as an insignificant pixel, and it may be flipped to black for embedding. The black pixel (marked with an arrow), which is actually a pseudo-insignificant pixel, would then be detected as an (false) insignificant pixel during blind detection. If $M$ is chosen to be equal or greater than 5 , the above possibilities of wrong detection would be avoided. Condition 3 is necessary due to the following reason: If after flipping, any pixel is generated among the 8-neighborhood 
pixels coming before in the scanning order and satisfy conditions 1 and 2, it could become a (false) insignificant pixel during detection; however, any pixel generated subsequently in the scanning order does not cause any error because of condition 2 .

We present the logical argument to show that with $M$ $=5$, no pixel in a block changes its status during blind detection without any tampering:

Case 1: Residual pixel Due to flipping of a single insignificant pixel, a residual pixel in its 8-neighborhood can change its status if it satisfies at least condition 1 and 2. If the residual pixel satisfies condition 1 and 2 due to flipping, it violates condition 3. During embedding, such a pixel (flipped one) would have been defined as a pseudo-insignificant pixel and not considered for flipping. When a residual pixel satisfies condition 2, not condition 1, during embedding, the following case may arise during detection. Due to multiple flipping of insignificant pixels, the residual pixel may satisfy condition 1. This is possible when multiple insignificant pixels are present in the 8-neighborhood of the residual pixel. With the choice of $M=5$ in condition 2, no residual pixel contains more than one insignificant pixel in its 8-neighborhood.

Case 2: Pseudo-insignificant pixel A pseudoinsignificant pixel $(P)$ will be converted to an insignificant pixel during blind detection if it satisfies condition 3 additionally. In Fig. 2, this is possible when no $(+)$ marked pixels satisfy conditions 1 and 2 after flipping the pixel $(P)$. During embedding, at least one $(+)$ marked pixel was found to satisfy condition 1 and 2 after flipping the pixel $(P)$.

During blind detection, the $(+)$ marked pixel may fail to satisfy condition 1 if there is flipping of an insignificant pixel in its 8-neighborhood. This possibility is avoided by the choice of $M=5$ in condition 2 . The $(+)$ marked pixel will continue to satisfy condition 2 , since residual pixels coming before it cannot change their status, as explained previously.

A pseudo-insignificant pixel $(P)$ will be converted to a residual pixel during blind detection if it does not satisfy any of conditions 1 and 2. Since there is no flipping in its $5 \times 5$ pixel neighborhood, it continues to satisfy condition 1 . It also continues to satisfy condition 2 , because any residual pixel coming before in scanning order does not change its status in the $5 \times 5$ pixel neighborhood.

Case 3: Insignificant pixel Since residual and pseudoinsignificant pixels do not change their status due to the flipping process, it is evident that insignificant pixels will satisfy conditions 1,2 , and 3 during blind detection.

\subsection{Embedding Erasable Watermark for Localization}

The proposed localization method is outlined in the following steps. The block diagram of the proposed method for embedding is shown in Fig. 4.

1. The whole image is divided into nonoverlapping

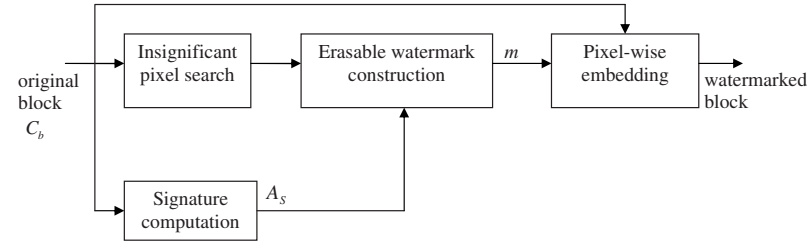

Fig. 4 Block diagram of embedding process in the proposed localization method.

blocks of $Y \times Z$ pixels. Watermarking is performed for each block independently.

2. In each block, an ordered set of insignificant pixels are searched in a sequential scanning order as described by conditions 1,2 and 3 in Sec. 2.2. The insignificant pixel set is losslessly compressed using the run-length coding scheme. Let the compressed data be denoted as $C_{D}$.

3. Authentication signature $A_{S}$ is computed from the block according to the following equation

$A_{S}=H\left(C_{b}, K, I_{b}, I_{K}\right)$,

where $H, C_{b}, K, I_{b}$, and $I_{K}$ denote hash function, current block in the original image, secret key, block index, and image index, respectively.

The block index is used in the computation of signature to resist block-swapping by a hostile attacker, and the image index is necessary to resist the Holliman-Memon attack. ${ }^{23}$

4. The compressed data and authentication signature are concatenated to create the message $m$, which is embedded in the insignificant pixel set producing the watermarked block. The embedding is performed pixel-wise, so an insignificant pixel holds one bit of $m$, and its pixel value is set equal to the signature bit it holds. Likewise, all blocks in the image are watermarked.

\subsection{Detecting Erasable Watermark for Localization}

The block diagram of the proposed method for detection is shown in Fig. 5.

1. To verify each block in the test image, the message $m^{d}$ is extracted by finding the insignificant pixel set in the embedding process. Its component pieces, the compressed version of insignificant pixel set $C_{D}^{d}$ and the authentication signature $A_{S}^{d}$ are extracted. The first 10 bits of $m^{d}$ represent the size of the compressed

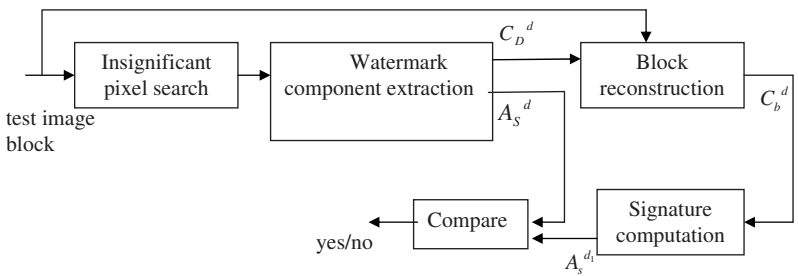

Fig. 5 Block diagram of blind detection process in the proposed localization method. 
The recent development of various methods of modulation such as PCM and PPM which exchange bandwidth for signal-to-noise ratio has intensified the interest in a general theory of communication. A basis for such a theory is contained in the important papers of Nyquist and Hartley on this subject. In the present paper we will extend the theory to include a number of new factors, in particular the effect of noise in the channel, and the savings possible due to the statistical structure of the original message and due to the nature of the final destination of the information.
The recent development of various methods of modulation such as $\mathrm{PCM}$ and $\mathrm{PPM}$ which exchange bandwidth for signal to-noise ratio has intensified the interest in a general theory of communication. A basis for such a theory is contained in the important papers of Nyquist and Hartley on this subject In the present paper we will extend the theory to include a number of new factors, in particular the effect of noise in the channel, and the savings possible due to the statistical structure of the original message and due to the nature of the final destination of the information. (a)

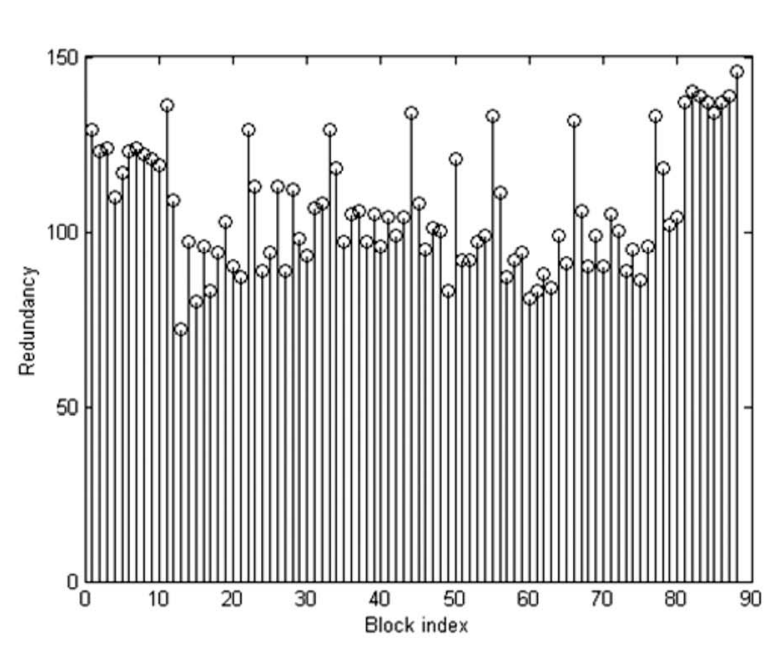

(c) (b)

Fig. 6 (a) Original text document image of size $320 \times 440$ pixels; (b) watermarked image after embedding the erasable watermark in each block; and (c) redundancy $(R)$ for each block of the original image.

data. While compressing the insignificant pixel set by run-length coding, a 10-bit representation is used for the number of white pixels and a 1-bit representation for the number of black pixels. This is because the possibility of occurrence of isolated pixels is less as compared to the background pixels. The first 10 bits giving the size information and the run-length encoded data represent the compressed data in $m^{d}$. The compressed version of the insignificant pixel set together with the current block is used to reconstruct the block $C_{b}^{d}$.

2. The authentication signature of the reconstructed block is computed as follows and compared with the extracted signature:

$A_{S}^{d_{1}}=H\left(C_{b}^{d}, K, I_{b}, I_{K}\right)$,

where $H, C_{b}^{d}, K, I_{b}$, and $I_{K}$ denote hash function, reconstructed block, secret key, block index, and image index, respectively.
3. If the signatures $A_{S}^{d}$ and $A_{S}^{d_{1}}$ match, then the reconstructed block is authentic. Verification of each block is performed independently to localize any tampering in the watermarked image.

\subsection{Results and Discussion}

In this section, we present simulation results by constructing the erasable watermark for the proposed block-wise localization method. The authentication signature to be used in this algorithm is the hashed message authentication code (HMAC). The HMAC is found by computing the one-way hash function of the data string that is a concatenation of the pixel set and secret key. In our method, high security against content modification is obtained by using the cryptographic hash function. We have implemented the message-digest (MD5) hash function ${ }^{24}$ to compute the HMAC. The output 128-bit HMAC is used as the authentication signature, and the message $m$ is constructed for each block as described in the proposed method. The value of $M$ is chosen to be 5 for achieving high watermark ca- 


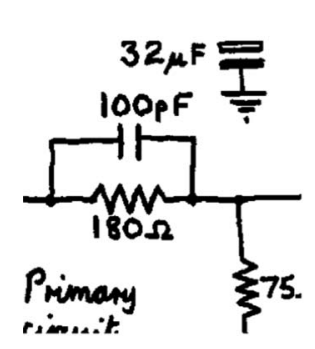

(a)

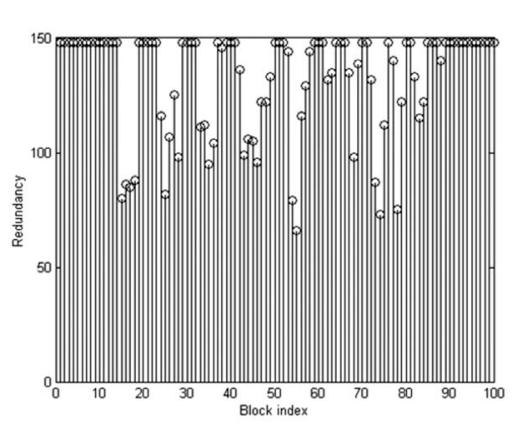

(c)

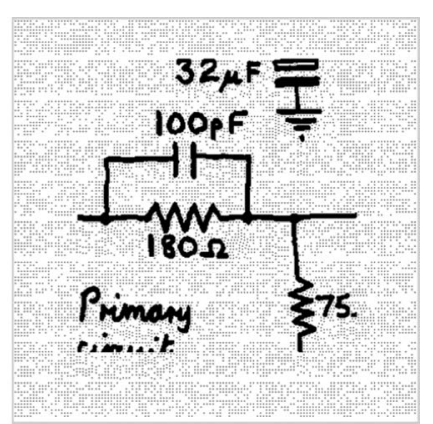

(b)
Fig. 7 (a) Original drawing image of size $400 \times 400$ pixels; (b) watermarked image after embedding the erasable watermark in each block; and (c) redundancy $(R)$ for each block of the original image.

pacity and correct watermark detection. We have chosen a block size of $40 \times 40$ pixels in our simulation, and the block size can be suitably modified if the length of the authentication signature is changed. In Figs. 6-8, the original and watermarked images are shown after the pixel-wise embedding of $m$ in each block. With no tampering, all blocks in the watermarked images are verified.

It is observed that even though background noise is present in the watermarked images, the content in the documents can be understood by the user. In the watermarked images, it is observed that the background noise appears to be random and different well-structured patterns can be recognized due to the inherent ability of human vision. The background noise carried by the watermark is very visible, even if the content can be interpreted by human users. Previous localization and restoration methods use perceptual models for reducing visual distortion in watermarked images. The proposed method is compared with the perceptual models such as the Princeton model $^{6}$ and the CWDD model. ${ }^{10}$ The evaluation of quality degradation in a binary document image is performed using the objective distortion measure. $^{25}$

The original images used in the experiment are shown in Fig. 9. A certain number of low-distortion pixels are flipped in an original image using the perceptual model. The proposed method is used to flip the same number of noise pixels in the original image. The image size and the number of flipped pixels for each original image are given in Table 1. A total of 36 images are generated and the objective distortion measure is computed for each of such images (Table 1). From the table, the distortion measure for an image generated using the proposed method is maximum in

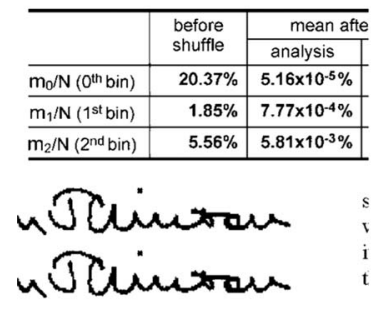

(a)

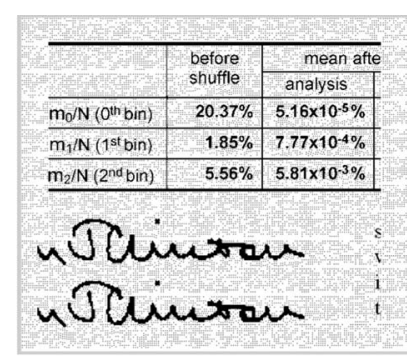

(b)

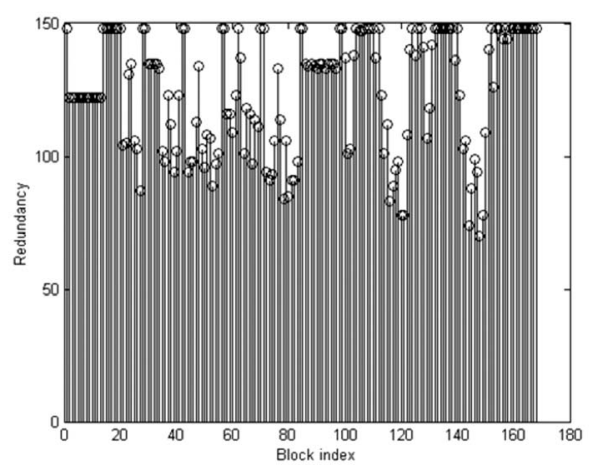

(c)

Fig. 8 (a) Original image of size $480 \times 560$ pixels containing text and signature; (b) watermarked image after embedding the erasable watermark in each block; and (c) redundancy $(R)$ for each block of the original image.

each case. This agrees with the fact that the noise pixels are very visible as compared to the low-distortion pixels chosen by the perceptual model. In the proposed method, a large number of noise pixels are flipped for embedding the erasable watermark. In such a case, the objective distortion measure will be significantly high. To illustrate, when 10,000 noise pixels are flipped in the original images, the objective distortion measure is 4.54, 4.78, 5.21, and 6.01, respectively. Such a large number of low-distortion pixels could not be flipped in the original images due to their lack of availability.

The noise pixels used for embedding the erasable watermark introduce quality degradation. In applications using document images, the authenticity of each block in the test image is first verified. If authenticity is verified, the exact copy of original image is restored, i.e., the watermark is removed. Since the reconstructed image is used for subsequent use and analysis, no application should suffer due to the quality degradation in the watermarked image.

For secure embedding, each block in the original image should have high watermark capacity. The capacity of a block is the number of bits that can be embedded within it. To analyze the performance of the proposed method in different images, we define the term redundancy $(R)$ in Eq. (3) as the number of bits available in a block to accommodate the signature,

\section{$R=$ size of the insignificant pixel set}

- compressed data size. 
any change made to any bit plane will be detected. The localization properties of this simple scheme can be improved if it is applied to image blocks rather than the whole image.

One of the first fragile watermarking techniques proposed for detection of image tampering was based on inserting check-sums of gray levels determined from the seven most significant bits into the least significant bits (LSB) of pseudo-randomly selected pixels [1]. In this paper, we are going to describe one possible implementation of this idea. First, we choose a large number $N$ that will be used for calculating the check sums. Its size directly influences the probability of making a change that might go undetected. The image is then divided into $8 \times 8$ blocks, and in each block, a different pseudo-random walk through all 64 pixels is generated. Let us denote the pixels as $p_{1}, p_{2}, \ldots$, $p_{64}$. We also generate 64 integers $a_{1}, a_{2}, \ldots, a_{64}$ comparable in size to $N$. The check sum $S$ is calculated as

(a)

The authors apply this technique to small $8 \times 8$ pixel blocks. The block is DCT transformed, and the frequency masking values $M(i, j)$ for each frequency bin $P(i, j)$ are calculated using a frequency masking model. The values $M\left(i_{2}\right)$ are the maximal changes that do not introduce perceptible distortions. The DCT coefficients are modified to $P_{S}(i j)$ according to the following expression

$P_{S}\left(i_{y}\right)=M\left(i_{y}\right)\left\{\left\lfloor P\left(i_{y}\right) / M\left(i_{y}\right)\right\rfloor+r\left(i_{y}\right) \operatorname{sign}\left(P\left(i_{y}\right)\right)\right\}$,

where $r\left(i_{2}\right)$ is a key-dependent noise signal in the interval $(0,1)$, and $\lfloor x\rfloor$ rounds $x$ towards zero. Since $\left|P(i, y)-P_{S}(i, j)\right| \leq$ $M(i, j)$, the modifications to DCT coefficients are imperceptible.

For a test image block with DCT coefficients $P_{S}(i, j)$, the masking values $M^{\prime}(i, j)$ are calculated. The error at $(i, j)$ is estimated by the following equation

$e^{\prime}=P_{S^{\prime}}-M\left\{r \operatorname{sign}\left(P_{S^{\prime}}\right)+\left\lfloor P_{S^{\prime}} / M^{\prime}-(r-1 / 2) \operatorname{sign}\left(P_{S^{\prime}}\right)\right\rfloor\right\}$,

(c)
There are some obvious advantages of this approach. First, the logo itself can carry some useful visual information about the image or its creator. It can also represent a particular authentication device or software. Second, by comparing the original logo with the recovered one, one can visually inspect the integrity of the image. Third, the authentication watermark is embedded not only in the LSBs of the image but somewhat deeper ( \pm 5 gay scales). This makes it more secure and harder to remove. Fourth, the method is fast, simple, and amenable to fast and cheap hardware implementation. This makes it very appealing for still image authentication in digital cameras.

This method, however, has a serious security gap if the same logo and key are reused for multiple images. Given two images $I_{1}$ and $I_{2}$ with gray levels $g^{(1)}$ and $g^{(2)}$ watermarked with the same key and $\log 0 L$, we have

$$
f_{g}\left(g^{(1)}\left(i_{j}\right)\right)=L\left(i_{2}\right)=f_{g}\left(g^{(2)}\left(i_{2}\right)\right) \text { for all }\left(i_{2}\right) \text {. }
$$

$$
\begin{aligned}
& Q_{\Delta l}(f)=0 \text { if }\left\lfloor f f\left(\Delta 2^{\prime}\right)\right\rfloor \text { is even, } \\
& Q_{\Delta l}(f)=1 \text { if }\lfloor f f(\Delta 2)\rfloor \text { is odd }
\end{aligned}
$$

at the quantization level $l$. If a wavelet coefficient $f_{k, I}(m, n)$ is chosen for watermark embedding, it is modified so that

$$
Q_{A, s}\left(f_{k, !}(m, n)\right)=w(i) \text { XOR } q k e y(m, n),
$$

where $w(i)$ is the $i$-th watermark bit and gkey is a bit generated from the image and a secret key. The construction of the quantization function $Q$ guarantees that one will never have to modify the coefficient at the level $l$ by more than $\pm \Delta 2$. The watermark is extracted by evaluating the expression

$$
w(i)=Q_{\Delta, s}\left(f_{k, l}^{\prime}(m, n)\right) \text { XOR qkey }(m, n),
$$

where $f^{\prime}$ is the wavelet coefficient of the potentially tampered image. The extent of tampering is evaluated using the number of correctly recovered watermark bits $w(i)$. The

(d)

Fig. 9 (a) to (d) The original images used for quality degradation comparison using the objective distortion measure.

A similar definition of redundancy has been used for natural images in Ref. 21 In Figs. 6, 7, and 8(c), it is shown that most blocks in the images have high redundancy for embedding $m$. Redundancy is an important parameter for performance evaluation of an erasable watermarking method because security, localization accuracy, and restoration capability depend on it. If redundancy in a block is too low, a sufficient number of signature bits cannot be embedded, leading to compromised security. To achieve sufficient redundancy, the block size needs to be increased, leading to a loss of localization accuracy. High redundancy of image blocks makes it possible to use a strong error correction coding scheme for effective restoration capability.

If $R$ in a block is less than 128 bits, e.g., 90 bits, then the first 90 bits of HMAC will be used to construct $m$ and authenticate the current block. Similarly, at the detector, the comparison between computed and extracted signature is performed only for the first 90 bits. In the proposed method, some blocks do not have sufficient redundancy to embed 128 bits. It is possible to increase redundancy in each block by increasing the block size; however, localiza- tion accuracy against tampering will be reduced. Thus, higher security can be achieved at the cost of localization accuracy.

If authenticity is verified, an exact copy of the original image is restored at the blind detector. To illustrate the localization capability of the proposed method, we perform the following modifications in the watermarked image in Fig. 7(b). The characters "Primary" at the left and bottom portion of the image are removed, and the attacked image is shown in Fig. 10. The detection is performed on the attacked image, and the detector correctly localizes the tampered blocks. Due to the use of a fragile watermark, it is not possible to recover the correct set of insignificant pixels in a block after any attack. The extracted and computed signatures do not match, and the block is declared to be tampered with or inauthentic. The proposed method is not robust to any kind of unintentional attacks, which is a limitation of fragile watermarking.

To test the effectiveness of our proposed method further, a total of 15 test images containing text, formulae, drawings, and tables are generated. In Tables $2-4, R_{\text {mean }}$ for each 
Table 1 Comparison of three methods (Princeton, CWDD, and proposed) with respect to quality degradation by using the objective distortion measure (Ref. 25).

\begin{tabular}{|c|c|c|c|c|c|}
\hline \multirow{2}{*}{$\begin{array}{l}\text { Original } \\
\text { image }\end{array}$} & \multirow[b]{2}{*}{ Size } & \multirow{2}{*}{$\begin{array}{l}\text { Number of } \\
\text { flipped } \\
\text { pixels }\end{array}$} & \multicolumn{3}{|c|}{ Objective distortion measure } \\
\hline & & & Princeton & CWDD & Proposed \\
\hline \multirow[t]{3}{*}{1} & $440 \times 520$ & 350 & 0.07 & 0.09 & 0.16 \\
\hline & & 550 & 0.11 & 0.14 & 0.25 \\
\hline & & 850 & 0.18 & 0.22 & 0.39 \\
\hline \multirow[t]{3}{*}{2} & $448 \times 512$ & 350 & 0.08 & 0.08 & 0.16 \\
\hline & & 550 & 0.12 & 0.14 & 0.25 \\
\hline & & 850 & 0.19 & 0.23 & 0.39 \\
\hline \multirow[t]{3}{*}{3} & $464 \times 512$ & 350 & 0.08 & 0.10 & 0.16 \\
\hline & & 550 & 0.13 & 0.16 & 0.25 \\
\hline & & 850 & 0.20 & 0.24 & 0.39 \\
\hline \multirow[t]{3}{*}{4} & $520 \times 544$ & 350 & 0.10 & 0.12 & 0.16 \\
\hline & & 550 & 0.15 & 0.19 & 0.25 \\
\hline & & 850 & 0.25 & 0.27 & 0.39 \\
\hline
\end{tabular}

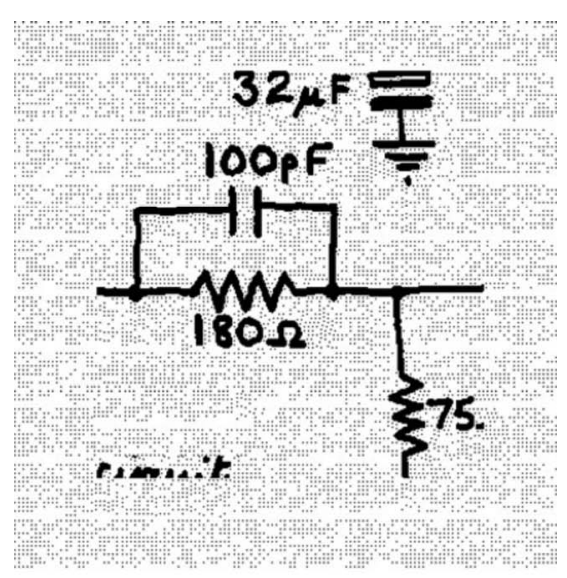

(a)

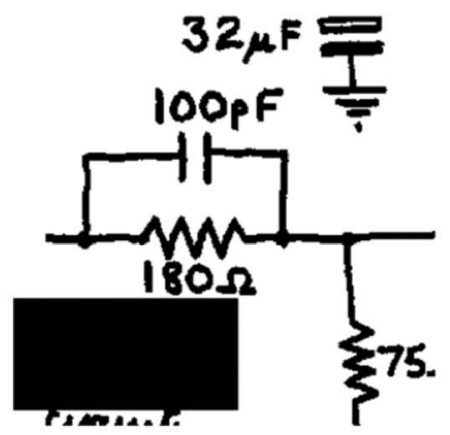

(b)

Fig. 10 (a) Attacked image. (b) Image showing the authentic reconstructed blocks; a total of 8 tampered blocks are shown in the dark region.
Table 2 Redundancy in test images with $36 \times 36$ pixel block size.

\begin{tabular}{lccc}
\hline \hline $\begin{array}{l}\text { Image } \\
\text { number }\end{array}$ & $\begin{array}{c}\text { Number of } \\
\text { blocks }\end{array}$ & $R_{\text {mean }}($ bits $)$ & $R_{\text {minimum }}$ \\
\hline 1 & 195 & 86.11 & 48 \\
2 & 195 & 87.16 & 48 \\
3 & 195 & 88.34 & 52 \\
4 & 195 & 92.25 & 53 \\
5 & 240 & 101.57 & 46 \\
6 & 224 & 100.60 & 62 \\
7 & 180 & 88.21 & 57 \\
8 & 368 & 97.82 & 61 \\
9 & 169 & 85.54 & 53 \\
10 & 238 & 86.45 & 52 \\
11 & 182 & 86.02 & 51 \\
12 & 208 & 84.16 & 55 \\
13 & 238 & 92.06 & 50 \\
14 & 238 & 92.54 & 50 \\
15 & 220 & 84.51 & 50 \\
\hline
\end{tabular}

test image is given for the block sizes of $36 \times 36,40 \times 40$, and $48 \times 48$ pixels, where $R_{\text {mean }}$ is the mean of redundancy of all the blocks in a test image. The minimum $R$ for the test images is reported in Tables 2-4. By taking the mean of the mean redundancy of all 15 test images, an approximate account of available watermark capacity in binary document images is obtained. The mean of the mean redundancy is found to be $90.22,113.27$, and 139.06 bits for $36 \times 36,40 \times 40$, and $48 \times 48$ pixels block size, respectively. Thus, it is shown that a hash output of 96 bits or 128 bits can be suitably embedded in blocks of various binary document images in the proposed method.

The performance of the proposed algorithm can be compared with the previous methods. ${ }^{18,19}$ The localization accuracy in the previous method ${ }^{18}$ was approximately at the block size of $128 \times 128$ pixels. In the proposed method, it has been improved to approximately the block size of 40 $\times 40$ pixels. The possibility of parity attack is not present in the proposed method because each message bit is embedded in an insignificant pixel instead of a block. For the proposed localization method, we discuss about two cases of false tamper detection. First, a block is declared to be inauthentic when there is no tampering in the block at all. The insignificant pixel positions are selected in such a manner that correct watermark detection is possible at the receiver without using the original image. When there is no tampering, each block in the test image is verified to be authentic. Second, a block is declared to be inauthentic when another block in the test image is tampered with. In 
Table 3 Redundancy in test images with $40 \times 40$ pixel block size.

\begin{tabular}{lccc}
\hline \hline $\begin{array}{l}\text { Image } \\
\text { number }\end{array}$ & $\begin{array}{c}\text { Number of } \\
\text { blocks }\end{array}$ & $R_{\text {mean }}(\mathrm{bits})$ & $R_{\text {minimum }}$ \\
\hline 1 & 168 & 111.95 & 67 \\
2 & 143 & 105.51 & 75 \\
3 & 156 & 110.82 & 73 \\
4 & 156 & 115.25 & 76 \\
5 & 182 & 123.19 & 78 \\
6 & 182 & 124.78 & 83 \\
7 & 154 & 114.31 & 82 \\
8 & 280 & 119.90 & 81 \\
9 & 144 & 110.02 & 72 \\
10 & 180 & 105.78 & 75 \\
11 & 144 & 107.56 & 66 \\
12 & 180 & 109.43 & 69 \\
13 & 192 & 115.88 & 75 \\
14 & 208 & 118.16 & 71 \\
15 & 180 & 106.52 & 71 \\
\hline \hline
\end{tabular}

the proposed method, watermarking is performed for each block independently; hence authenticity status of each block remains unaffected by the attacker's activity within other blocks. Thus, there is no possibility of false tamper detection and incorrect localization in the proposed method.

\section{Proposed Restoration Method in Text Document Images}

\subsection{Basic Concept}

After localization of tampered regions in a document image, the next relevant question arises about the possibility of restoring the modified portions. In this section, a new method to address the issue of text sequence restoration is proposed using erasable watermarks in conjunction with error correction coding. The method is particularly effective for text document images in electronic form. In the proposed method, embedding an erasable watermark in each block of an image will introduce visible noise in watermarked images that can be made available for different users. The relevant information contained in the text document is preserved so that the user can understand the documents. The user can localize any tampering to the watermarked image with high probability and accuracy. After localization, the original text sequence can be restored by using error correction coding techniques. The watermark can then be erased from the authenticated blocks to retrieve the distortion-free original image for further analysis and application.
Table 4 Redundancy in test images with $44 \times 44$ pixel block size.

\begin{tabular}{|c|c|c|c|}
\hline $\begin{array}{l}\text { Image } \\
\text { number }\end{array}$ & $\begin{array}{c}\text { Number of } \\
\text { blocks }\end{array}$ & $R_{\text {mean }}(\mathrm{bits})$ & $R_{\text {minimum }}$ \\
\hline 1 & 143 & 136.48 & 89 \\
\hline 2 & 120 & 130.25 & 99 \\
\hline 3 & 132 & 136.75 & 104 \\
\hline 4 & 132 & 141.90 & 106 \\
\hline 5 & 156 & 151.51 & 109 \\
\hline 6 & 156 & 152.14 & 111 \\
\hline 7 & 130 & 142.32 & 109 \\
\hline 8 & 247 & 148.81 & 99 \\
\hline 9 & 121 & 136.19 & 93 \\
\hline 10 & 154 & 132.43 & 90 \\
\hline 11 & 121 & 133.26 & 95 \\
\hline 12 & 143 & 132.24 & 92 \\
\hline 13 & 154 & 140.29 & 91 \\
\hline 14 & 168 & 142.76 & 102 \\
\hline 15 & 144 & 128.63 & 100 \\
\hline
\end{tabular}

\subsection{Finding Insignificant Pixels after Preprocessing}

For restoration applications, a substantial amount of watermark capacity is required to embed necessary information bits. The availability of high capacity through constructing an erasable watermark is illustrated in Sec. 2.5. In our investigation, we found that it is necessary to further increase the available watermark capacity for achieving effective restoration capability. The procedure to find insignificant pixels in a block is described in Sec. 2.2. For correct and blind detection, it was shown that the minimum value of $M$ should be chosen as 5. To increase the redundancy $R$ in a block, the value of $M$ should be decreased such that a greater number of insignificant pixels could be found. At the same time, to ensure correct watermark detection, certain modifications are necessary in the watermarking process. If the value of $M$ is chosen to be 3 , then correct detection is not possible due to the following reasons: As shown in Fig. 3(b), there is a possibility of false insignificant pixel generation after flipping two black insignificant pixels. In Fig. 3(c), flipping of a white pixel (marked 2) could convert a pseudo-insignificant pixel (marked by an arrow) to an insignificant pixel. To avoid such possibilities of wrong detection, we modify the procedure for finding the insignificant pixels after performing a preprocessing step. If two isolated pixels are found within a $3 \times 3$ pixel window centered on any pixel, one of them will be flipped to become a white pixel. Isolated pixels in a text document image do not carry significant information and the probability is low in finding two isolated pixels in a $3 \times 3$ pixel 


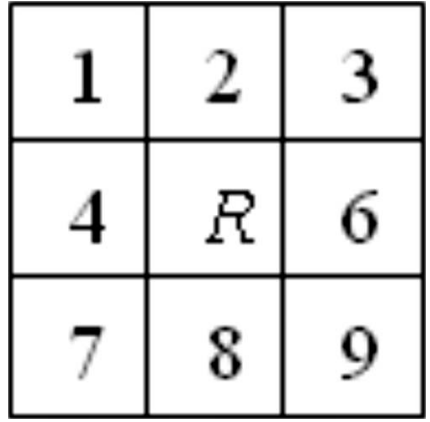

Fig. 11 The residual pixel $R$ with its 8-neighborhood to analyze multiple insignificant pixel flipping.

window. Thus flipping such isolated pixels does not have much impact on the text document content. After this preprocessing step, an ordered set of insignificant pixels is searched in a sequential scanning order starting from left to right and top to bottom. A pixel within a block of the preprocessed image is defined to be an insignificant pixel if the following three conditions are satisfied. A pixel is defined as a pseudo-insignificant pixel if it satisfies conditions 1 and 2 but does not satisfy condition 3 . The rest of the pixels are defined as residual pixels.

Condition 1 The pixel is either a background pixel or an isolated pixel.

Condition 2 In a $3 \times 3$ pixel window centered on the current pixel, there should not be any insignificant pixel, and in a $5 \times 5$ pixel window, there should not be any pseudo-insignificant pixel already found in the block.

Condition 3 After flipping the current pixel, there

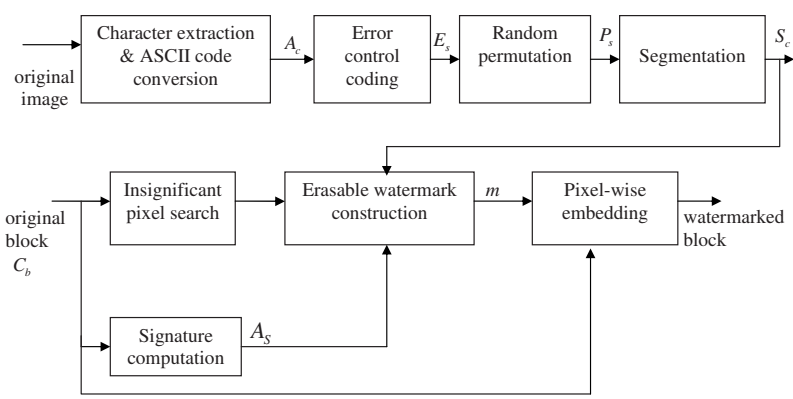

(a)

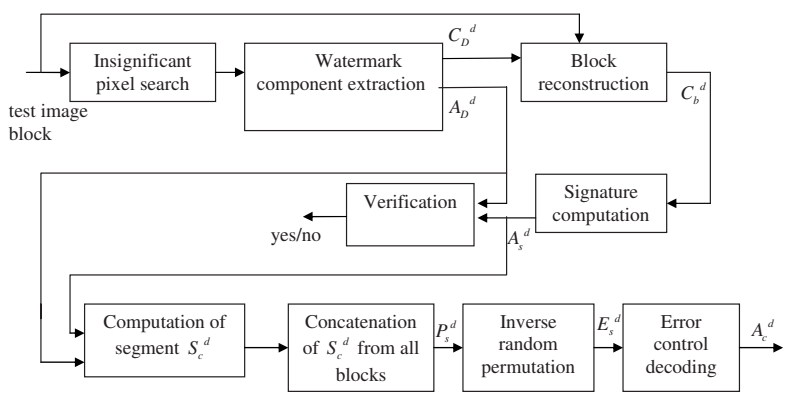

(b)

Fig. 12 Block diagram of the proposed restoration method: (a) Embedding process; and (b) blind detection process. should not be any pixel in its 8-pixel neighborhood that comes before in the scanning order and satisfies the preceding two conditions.

We present a logical argument to show that modification of condition 2 along with a preprocessing step will ensure correct blind detection in restoration:

Case 1: Residual pixel As discussed in Sec. 2.2, a residual pixel could change its status due to multiple insignificant pixels in its 8-neighborhood. This is possible when a residual pixel satisfies condition 2, not condition 1, during embedding. In Fig. 11, we analyze the 8-neighborhood of a residual pixel containing multiple insignificant pixels, when $M=3$. The center pixel $R$ is a residual pixel and the pixels (1 to 4 ) come before it in scanning order. The pixels (6 to 9 ) can be considered as insignificant pixels. However, the pixels (1 to 4 ) are not considered as insignificant pixels for the sake of analysis, because $R$ will then never change its status due to condition 2 . We also consider the pixels ( 1 to 4 ) as white pixels so that $R$ can possibly satisfy condition 1 due to multiple insignificant pixels.

Due to the $3 \times 3$ pixel neighborhood constraint in condition 2 , there can be a maximum of two insignificant pixels among pixels (6 to 9). This occurs when pixel (7) and either of pixel (6) or (9) are insignificant pixels. We examine different cases of insignificant pix-

The recent development of various methods of modulation such as PCM and PPM which exchange bandwidth for signal-to-noise ratio has intensified the interest in a general theory of communication. A basis for such a theory is contained in the important papers of Nyquist and Hartley on this subject. In the present paper we will extend the theory to include a number of new factors, in particular the effect of noise in the channel, and the savings possible due to the statistical structure of the original message and due to the nature of the final destination of the information

(a)

The recent development of various methods of modulation such as PCM and PPM which exchange bandwidth for signal to noise ratio has intensified the interest in a general theory of communication. A basis for such a theory is contained in the important papers of Nyquist and Hartley on this subject In the present paper we will extend the theory to include a number of new factors, in particular the effect of noise in the channel, and the savings possible due to the statistical structure of the orignal message and due to the nature of the final destination of the information

(b)

Fig. 13 (a) Original image of $320 \times 440$ pixels; and (b) the watermarked image after embedding the message $m$ in 110 blocks. 


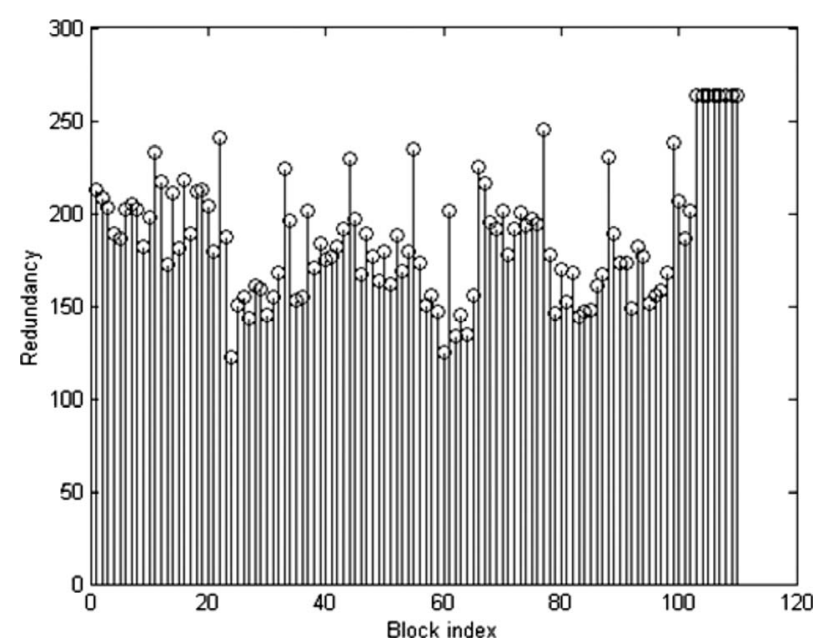

Fig. 14 Redundancy $(R)$ for each block of the original image.

els: (1) one isolated and another background pixel, (2) both isolated pixels, and (3) both background pixels:

- (7) Isolated pixel; (6) or (9) background pixel. Pixel $R$ will satisfy condition 1 when pixel (7) is flipped and pixel (6) or (9) remains unchanged. However, this case is not possible since pixel (7) would be declared as pseudo-insignificant during embedding.

- (7) background pixel; (6) or (9) isolated pixel. This case is identical to the previous one. Pixel $R$ will satisfy condition 1 when pixel (7) remains unchanged and pixel (6) or (9) is flipped. However, this case is not possible since pixel (6) or (9) would be declared as pseudo-insignificant during embedding.

- (7) Isolated pixel; (6) or (9) isolated pixel. Pixel $R$ will satisfy condition 1 when both pixels are flipped. However, this case is not possible due to the preprocessing step before embedding.

- (7) background pixel; (6) or (9) background pixel. Pixel $R$ will satisfy condition 1 when both pixels remain unchanged. This implies that $R$ satisfied condi-

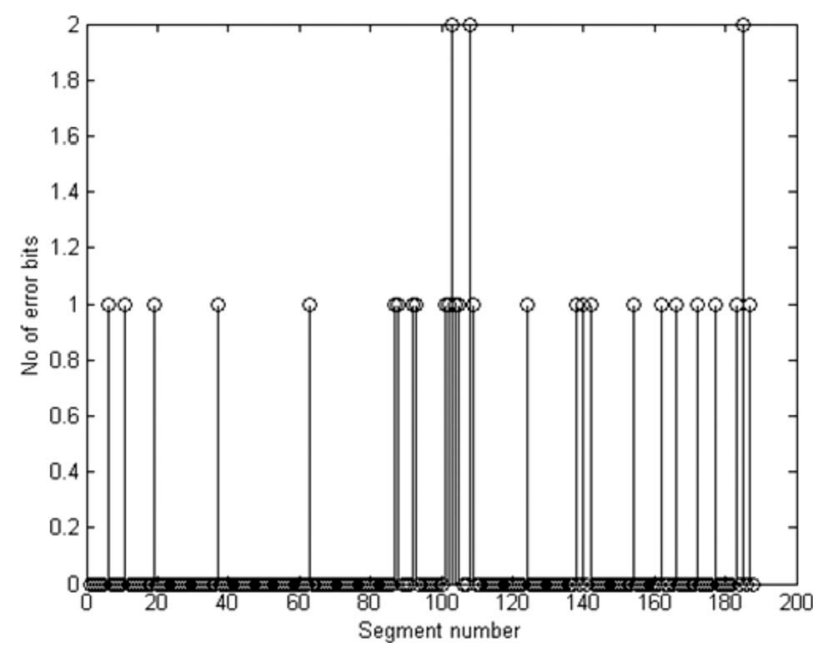

Fig. 15 The number of error bits in each 63-bit segment of the bit sequence $E_{s}^{d}$ extracted from the watermarked image.

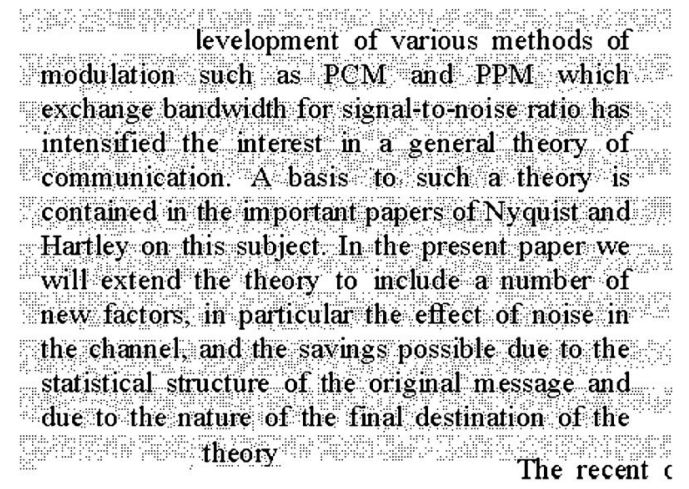

Fig. 16 Attacked image after multiple alterations like text deletion, insertion, substitution, and block swapping.

tion 1 but not condition 2 during embedding. Otherwise, it would have been declared at least as a pseudoinsignificant pixel. In that case, an insignificant pixel could not be present in its 8-neighborhood.

Case 2: Pseudo-insignificant pixel Since $M=5$ is used in condition 2 for pseudo-insignificant pixels, the logical argument given in the case of localization holds good for restoration.

Case 3: Insignificant pixel Since residual and pseudoinsignificant pixels do not change their status due to the flipping process, it is evident that all insignificant pixels will satisfy conditions 1,2 , and 3 during blind detection.

\subsection{Erasable Watermark Embedding for Restoration}

The block diagram of the proposed restoration method is shown in Fig. 12. We outline the proposed restoration method in the following steps.

1. The original image is divided into nonoverlapping blocks of $32 \times 40$ pixels. The size of the original image is chosen to be the multiple of individual block size. Watermarking is performed for each block independently.

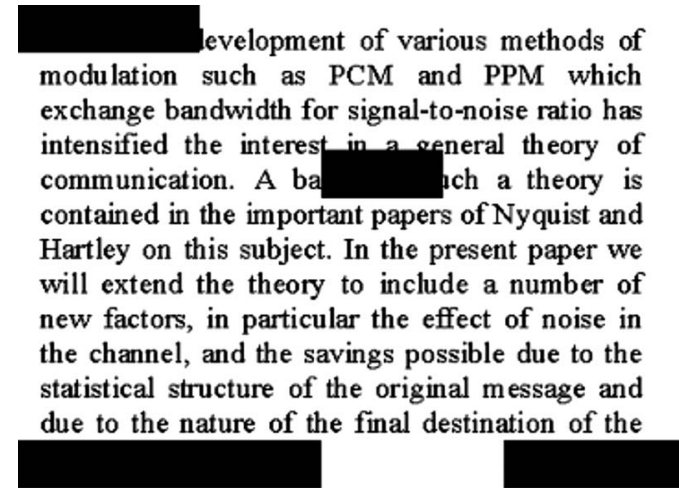

Fig. 17 Image showing the authentic reconstructed blocks; a total of 13 tampered blocks are shown in the dark regions. 


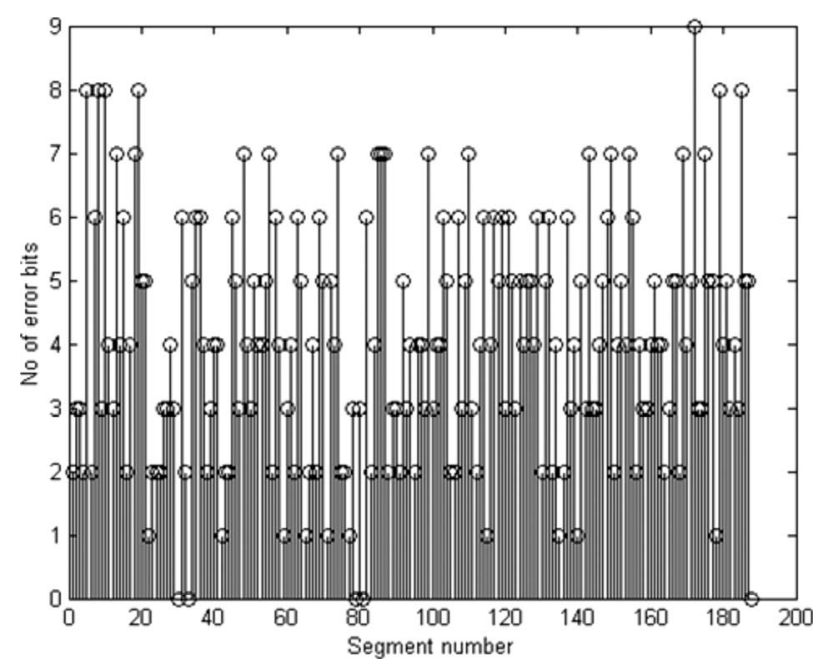

Fig. 18 The number of error bits in each 63-bit segment of the bit sequence $E_{s}^{d}$ extracted from the attacked image.

2. The text sequence in the original image is extracted in a sequential order, and a binary sequence $A_{c}$ is obtained after each character is converted to the corresponding ASCII code.

3. Another binary sequence $E_{s}$ is obtained by encoding $A_{c}$ with an $[n, k]$ Bose, Ray-Chaudhuri, Hocquenghem $(\mathrm{BCH})$ encoder. ${ }^{26}$ Using this scheme, $t_{s}$ error bits can be corrected in an $n$-bit ECC encoded segment. Before ECC encoding, zero-padding can be performed such that the number of bits in the sequence $A_{c}$ becomes an integral multiple of $k$. The parameters $n$ and $k$ are chosen such that the total number of bits in $E_{s}$ is less than or equal to $108 \times B$, where $B$ is the total number of blocks in the image. The number 108 is chosen here because a maximum of 108 bits in $E_{s}$ will be embedded in a single block, as described in the following steps.

4. After ECC encoding, zero-padding can be performed in the sequence $E_{s}$ to make the number of bits exactly equal to $108 \times B . E_{S}$ is then randomly permuted using the secret key $K$. Let the permuted sequence be denoted as $P_{s}$. The errors due to tampering in a localized region of the image often come as bursts. The random permutation distributes the burst type of error over the whole image; thus increasing the correction capability of ECC coding.

5. The sequence $P_{s}$ is divided into $B$ nonoverlapping segments of size 108 bits each. Let each such segment be denoted by $S_{c}$. A maximum of 108 bits of a particular segment $S_{c}$ is used in the embedding process of the corresponding block in the sequential order.

6. In each block, an ordered set of insignificant pixels are searched in the sequential scanning order by using conditions 1,2 , and 3 described in Sec. 3.2. The insignificant pixel set is losslessly compressed using the run-length coding scheme. A total of 10 bits are used to represent the size of the run-length encoded data. These 10 bits along with the run-length encoded data represent the compressed data $C_{D}$. The term re-

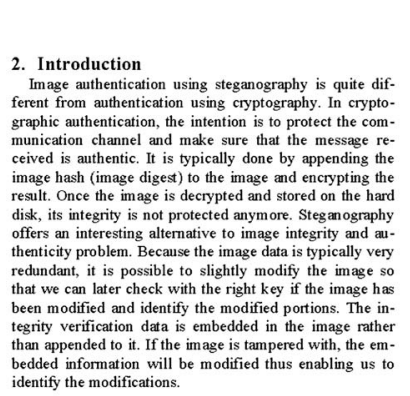

(a)

algorithm in which a data bit ' 1 ' is embedded if the percentage of white pixels was greater than a given threshold, and a data bit ' $\theta$ ' is embedded if the percentage of white pixels was less than another given threshold. This algorithm was not rob
hiding capacity was low.

Wu et al 8 hid data in a binary image using a ele hid dat in a binay inage using a hierarchical model in which human perception was taken into consideration. Distortion that occurred due to flipping of a pixel was ceasured by constleng the change in smoothness and connectiviy of a las window crat mumber of bick pivels to be either odd or otal number of black pixels to be either odd or

(c)

The use of a suitable perceptual model is necessary to minimize the visual distortion in the marked images, because minor modification to the pixels can be perceptible since the pixels are either black or white In this paper, a new perceptual model is proposed for binary imes appications. In our model, the distoris applications. In our model, the distortion that occurs after flipping a pixel is estimated on the novel curvatureweighted distance difference (CWDD) measure between two contour segments. Through subjective tests the perceptual measure is validated and highly correlated with human perception.

(b)

\section{REFERENCES}

[1] S. H. Low, N. F. Maxemchuk, and A. M. Lapone, "Document identification for copyright protection using centroid detection," IEEE Trans. on Communication, vol. 46, no. 3, March 1998, pp. 372-383.

[2] S. H. Low, and N. F. Maxemchuk, "Performance comparison of two text marking methods," IEEE Journal on Selected Areas in Communications, vol. 16, no. 4, May 1998.

[3] E. Koch, J. Zhao, "Embedding robust labels into images for copyright protection, "Proc. International Congress protection, "Proc. International Congress on Intellectual Property Rights for

Fig. 19 (a) to (d) The original images used for showing restoration capability of the proposed method.

dundancy $(R)$ is computed according to Eq. (3).

7. Let the sets $S_{1}$ and $S_{2}$ be defined such that $S_{1}$ contains the first 32 bits and $S_{2}$ contains the next $n_{s}$ bits of the segment $S_{c}$, where

$n_{s}=\min (R-64,76)$.

In Eq. (4), $n_{s}$ is defined to adjust the size of embedded data if the current block has insufficient redundancy.

8. The 64-bit authentication signature $A_{s}$ defined by

$A_{s}=H\left(C_{b}, K, I_{b}, I_{K}, S_{2}\right)$,

is computed from the current block, where $H, C_{b}, I_{b}$, and $I_{K}$ denote hash function, current block in the original image, block index, and image index, respectively.

The reason for using $S_{2}$ in signature computation is to secure the embedded information against hostile attacks. If these bits used for restoration purpose are altered by the attacker in a block, then the block becomes inauthentic.

9. The authentication data $A_{D}$ of size $\left(n_{s}+64\right)$ bits consists of three sets. Let the sets be denoted as $A_{D}^{1}, A_{D}^{2}$, and $A_{D}^{3}$, where $A_{D}^{1}$ contains the first 32 bits, $A_{D}^{2}$ contains the next 32 bits, and $A_{D}^{3}$ contains the remaining $n_{s}$ bits of $A_{D}$. The three sets are computed according to Eq. (6):

$A_{D}^{1}=A_{s}^{1} \oplus S_{1}$, 


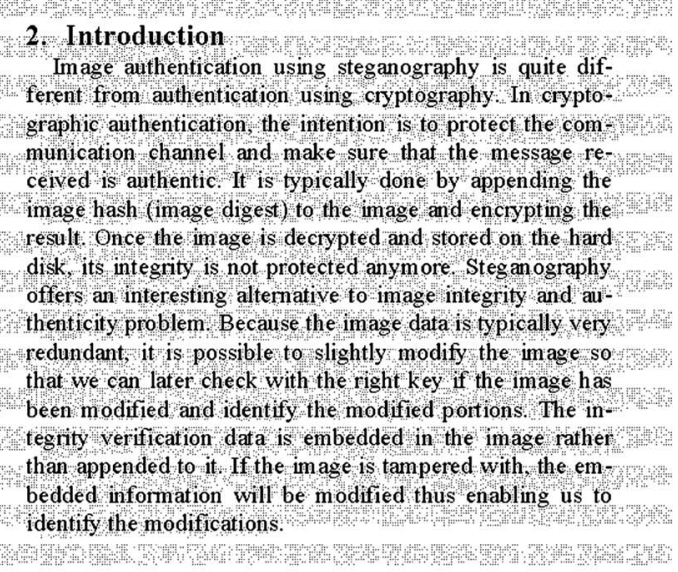

(a)
The use of a suitable perceptual model is necessary to minimize the visual distortion in the marked images, because minor modification to the pixels can be perceptible since the pixels are either black or white. In this paper, a new perceptual model is proposed for binary images that is useful for data hiding applications. In our model, the distortion that occurs after flipping a pixel is estimated on the novel curvatureweighted distance difference (CWDD) measure between two contour segments. Through subjective tests the perceptual measure is validated and highly correlated with human perception.

\begin{abstract}
algorithm in which a data bit $T$ is embedded if the percentage of white pixels was greater than a given threshold and a data bit $\theta^{\prime}$ is embedded if the percentage of white pixels was less than another given threshold This algorithm was not robust to attacks and the hiding capacity was low.

-

Wu et al /8/ hid data in a binary image using a hierarchical model in which human perception was taken into consideration. Distortion that occurred due to lipping of a pixel was measured by considering the change in smoothness and connectivity of a $3 x 3$ window centered at the pixel. In a block modiffing the fotal number of black pixels to be either odd or: even embeds data bits. Ln et al proposed an What
\end{abstract}

(b)

(c)

\section{REFERENCES}

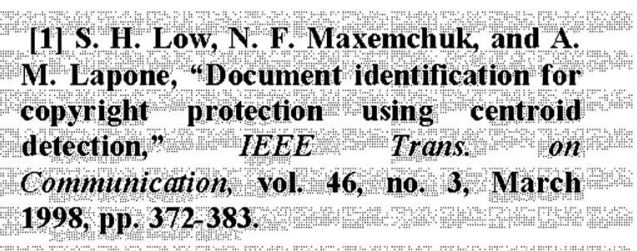

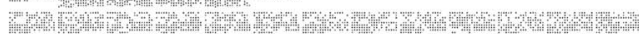

[2] S. H. Low, and N. F. Maxemchuk, "Performance comparison of two text marking methods, IEEE Journal on Selected Areas in Communications, vol. 16, no. 4, May 1998.

[3] E. Koch, J. Zhao, "Embedding robust labels into images for copyright protection, 9 Proc. International Congress on Intellectual Property Rights for Specialized Information, Knowledge \&

Fig. 20 (a) to (d) The corresponding watermarked images.

$A_{D}^{2}=A_{s}^{2} \oplus S_{1}^{c}$

$A_{D}^{3}=S_{2}$,

where $\oplus$ is the exclusive OR operation, $A_{s}^{1}$ contains the first 32 bits, and $A_{s}^{2}$ contains the remaining 32 bits of $A_{s}$.

In Eq. (6), the same 32 bits of $S_{1}$ are used in the first two segments for tamper localization. During blind detection on an attacked image, the tampered block will not satisfy Eq. (10) due to the use of a fragile watermark. If some bits of $S_{2}$ are used in the second part, it is difficult to establish a relationship between two segments in Eq. (10). Thus, tamper localization will not be possible after attacks.

10.The compressed data $C_{D}$ and authentication data $A_{D}$ are concatenated to create the message $m$, which is embedded in the insignificant pixel set. The embed- ding is performed pixel-wise, so an insignificant pixel holds one bit of $m$ and its pixel value is set equal to the message bit it holds. According to Eq. (4), the maximum size of $S_{2}$ is 76 bits. Thus the maximum size of $A_{D}$ in a block is equal to 140 bits. In an ideal case, each block of the original image should have $R \geqslant 140$.

\subsection{Erasable Watermark Detection for Restoration}

1. The test image is divided into nonoverlapping blocks of $32 \times 40$ pixels. Verification is performed for each block independently.

2. To verify each block, the message $m$ is extracted by finding the insignificant pixel set using conditions 1, 2, and 3 as described in Sec. 3.2. During detection, it was found that preprocessing was not necessary. The parameters $R$ and $n_{s}$ are computed using Eqs. (3) and 


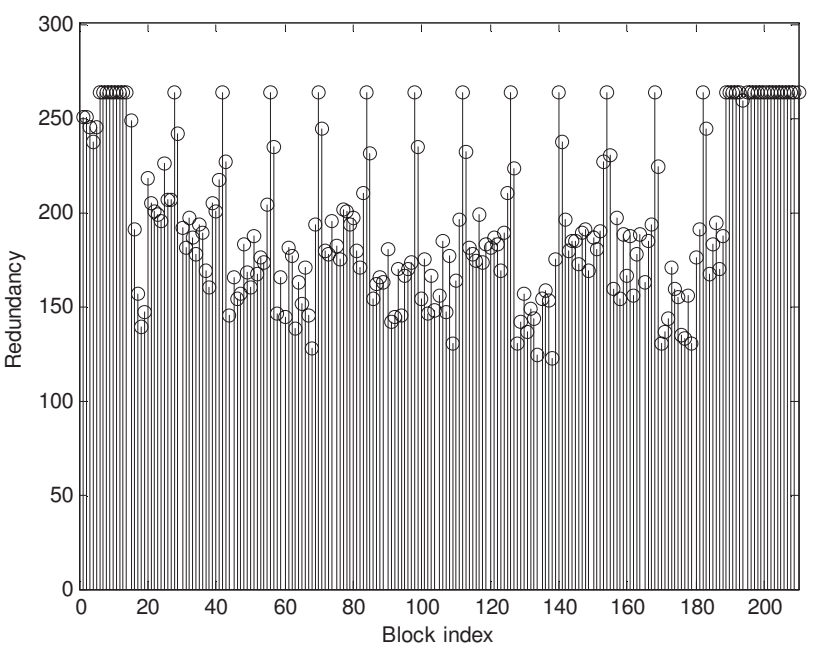

(a)

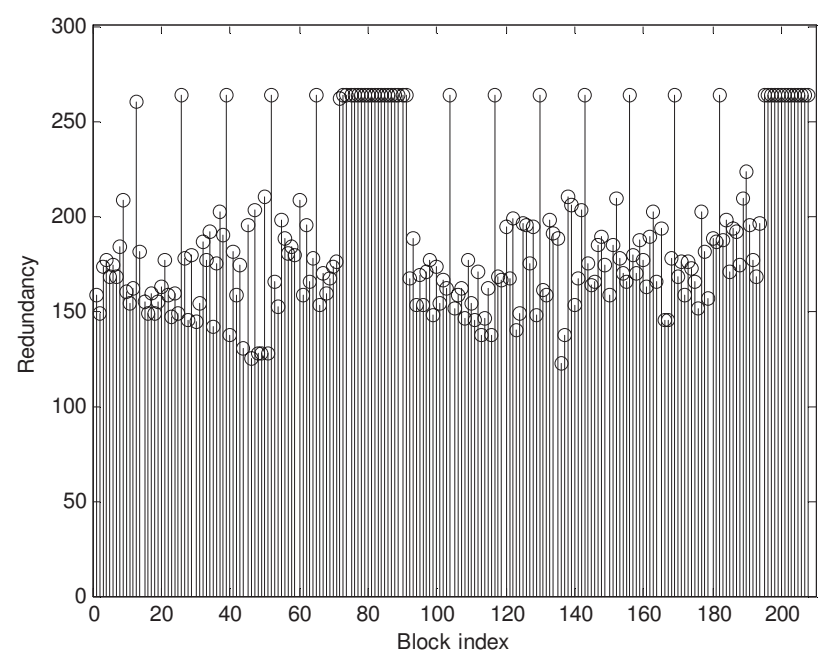

(c)

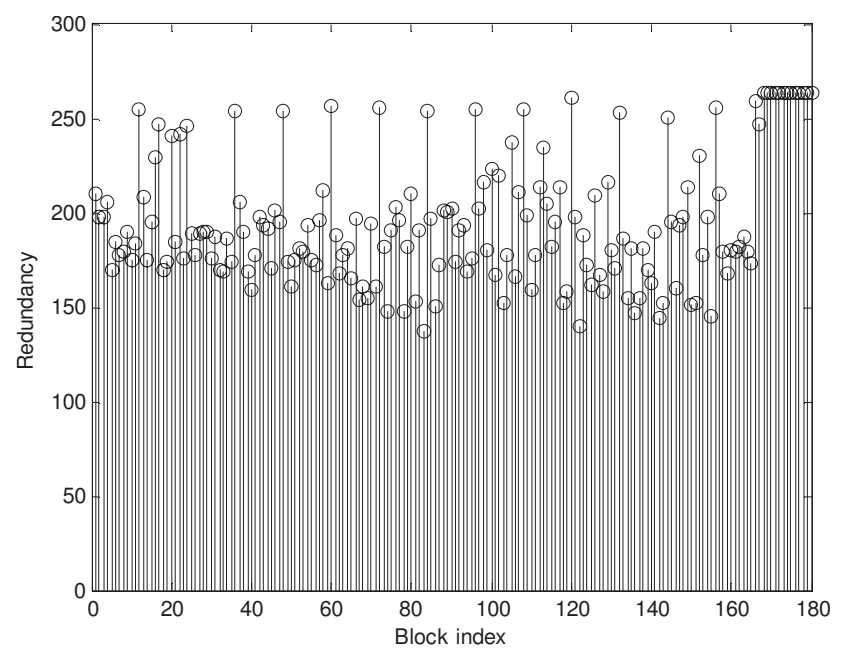

(b)

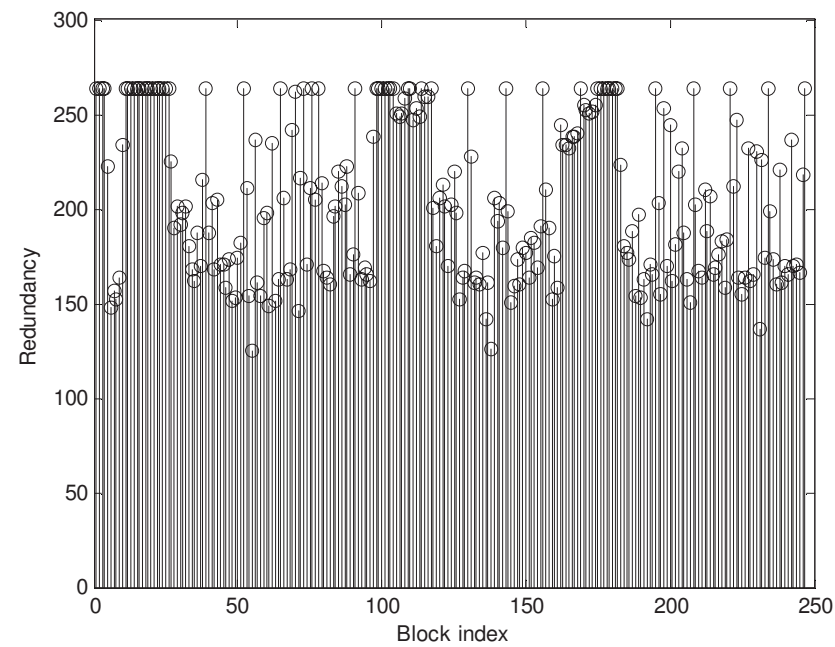

(d)

Fig. 21 (a) to (d) Redundancy $(R)$ for each block of the original images.

(4), respectively. The compressed data $C_{D}^{d}$ and the authentication data $A_{D}^{d}$ are extracted from the message $m$. The compressed data $C_{D}^{d}$ together with the current watermarked block is used to reconstruct the block $C_{b}^{d}$.

3. The authentication data $A_{D}^{d}$ of size $\left(n_{s}+64\right)$ bits is separated into three sets. Let the sets be denoted as $A_{D}^{a}, A_{D}^{b}$, and $A_{D}^{c}$, where $A_{D}^{a}$ contains the first 32 bits, $A_{D}^{b}$ contains the next 32 bits, and $A_{D}^{c}$ contains the remaining $n_{s}$ bits of $A_{D}^{d}$.

4. The 64-bit signature $A_{s}^{d}$ is computed from the reconstructed block $C_{b}^{d}$ according to

$A_{s}^{d}=H\left(C_{b}^{d}, K, I_{b}, I_{K}, A_{D}^{c}\right)$,

where the two sets $A_{s}^{a}$ and $A_{s}^{b}$ are defined such that $A_{s}^{a}$ contains the first 32 bits, and $A_{s}^{b}$ contains the remaining 32 bits of $A_{s}^{d}$.
5. The segment $S_{c}^{d}$ of size $\left(n_{s}+32\right)$ bits is computed, which contains information bits for restoration. It consists of two sets: $S_{1}^{d}$ contains the first 32 bits, which is computed

$S_{1}^{d}=A_{D}^{a} \oplus A_{s}^{a}$.

The other set $S_{2}^{d}$ contains the remaining $n_{s}$ bits and is equal to $A_{D}^{c}$.

6. Another set $S_{1}^{d_{1}}$ is computed according to

$S_{1}^{d_{1}}=A_{D}^{b} \oplus A_{s}^{b}$.

The reconstructed block $C_{b}^{d}$ is authentic, only if the following condition:

$S_{1}^{d}=\left(S_{1}^{d_{1}}\right)^{c}$,

is satisfied. 


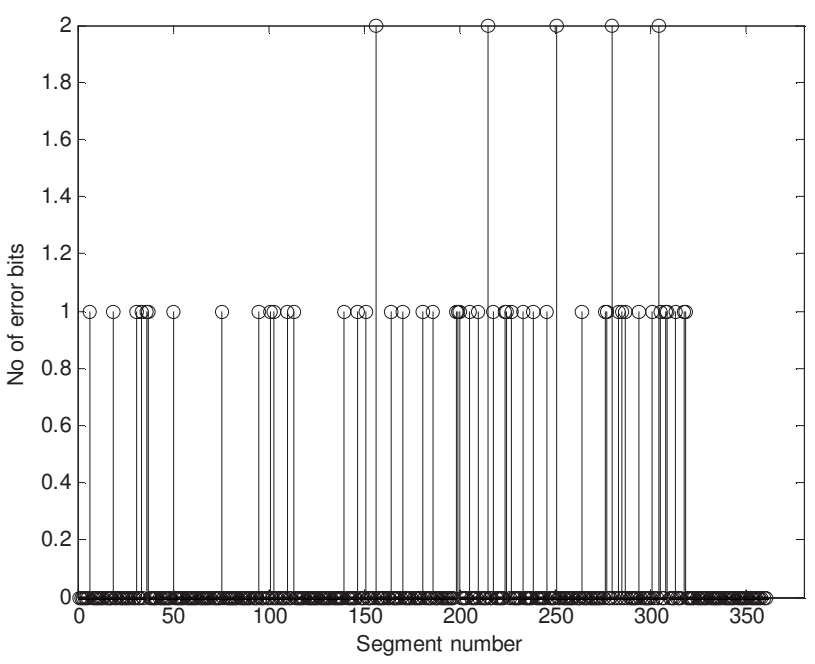

(a)

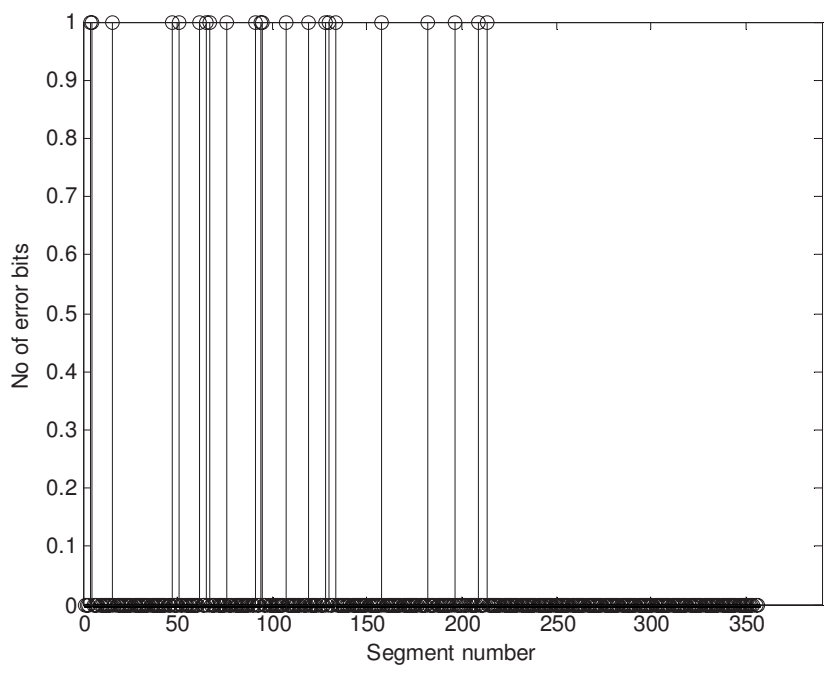

(c)

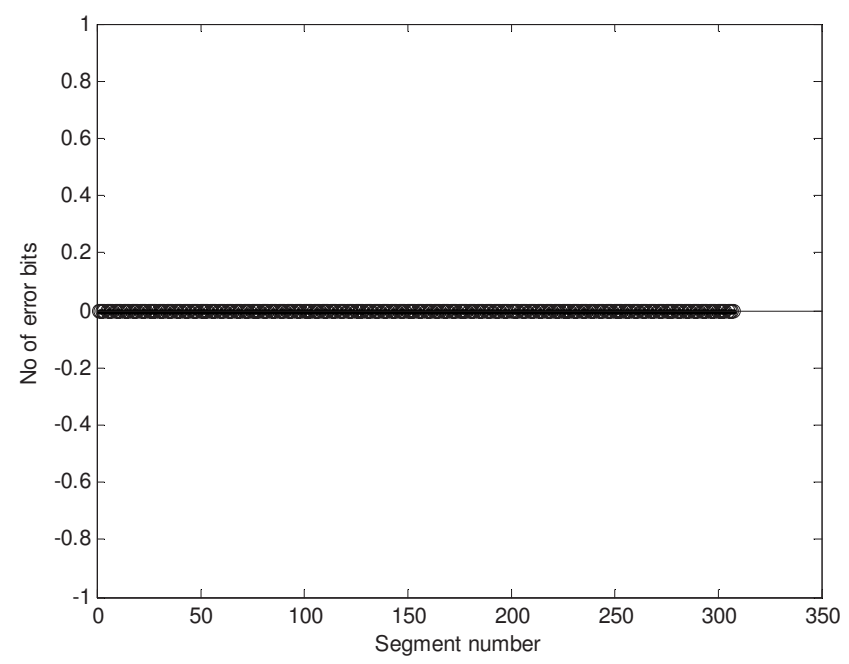

(b)

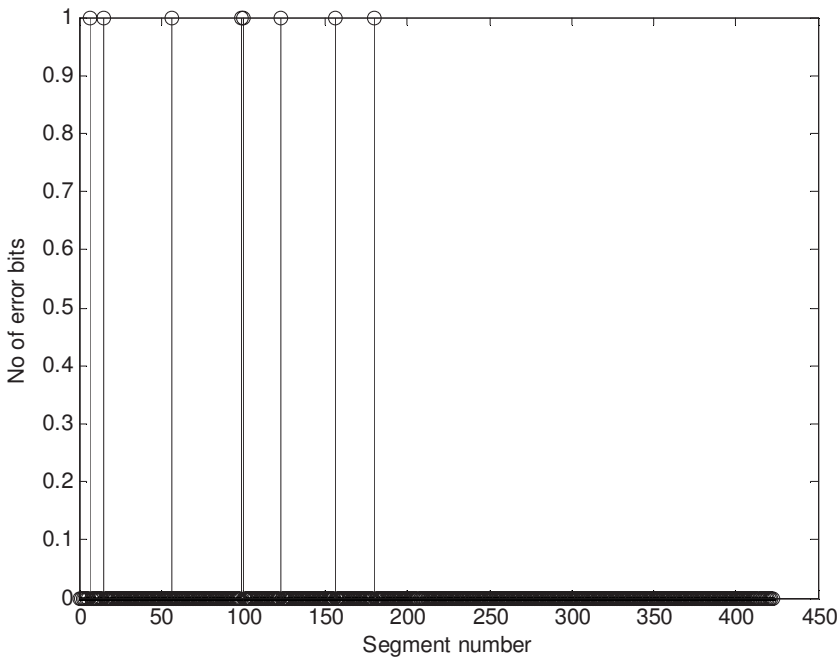

(d)

Fig. 22 (a) to (d) The number of error bits in each 63-bit segment of the bit sequence $E_{s}^{d}$ extracted from the watermarked images.

7. If necessary, zero-padding is performed such that the size of $S_{c}^{d}$ becomes equal to 108 bits. During verification of all blocks in the sequential order, the segments $S_{c}^{d}$ are concatenated to obtain the sequence $P_{s}^{d}$.

8. The sequence $E_{s}^{d}$ is obtained after inverse permutation of $P_{s}^{d}$ by using the secret key $K$. The $[n, k] \mathrm{BCH}$ decoder is applied in each $n$-bit segment of $E_{s}^{d}$ to obtain $A_{c}^{d}$. If the number of error bits in $E_{s}^{d}$ (with respect to $E_{s}$ ) is within the correction capability of ECC decoder, the original text sequence can be correctly extracted from $A_{c}^{d}$.

\subsection{Results and Discussion}

In this section, we present simulation results by constructing the erasable watermark using the proposed restoration method. The authentication signature to be used in this method is the hashed message authentication code (HMAC). The 64-bit HMAC is used as the authentication signature, and the message $m$ is constructed for all blocks as described in the proposed method. The length of HMAC is reduced to 64 bit from 128 bits to accommodate extra information bits for restoration. A sufficient number of insignificant pixels are available within a block size of 32 $\times 40$ pixels. The choice of higher block size will produce higher capacity; however, the number of error bits contributed by a tampered block is increased. Moreover, restoration would be affected.

The values of $n$ and $k$ are chosen to be 63 and 18, respectively for $\mathrm{BCH}$ error correction coding. Using this scheme, a total of 10 error bits can be corrected in a 63-bit ECC encoded segment. The use of $[63,18] \mathrm{BCH}$ code is found to be a conservative choice, since it is not document dependent. For effective restoration, the strength of error 


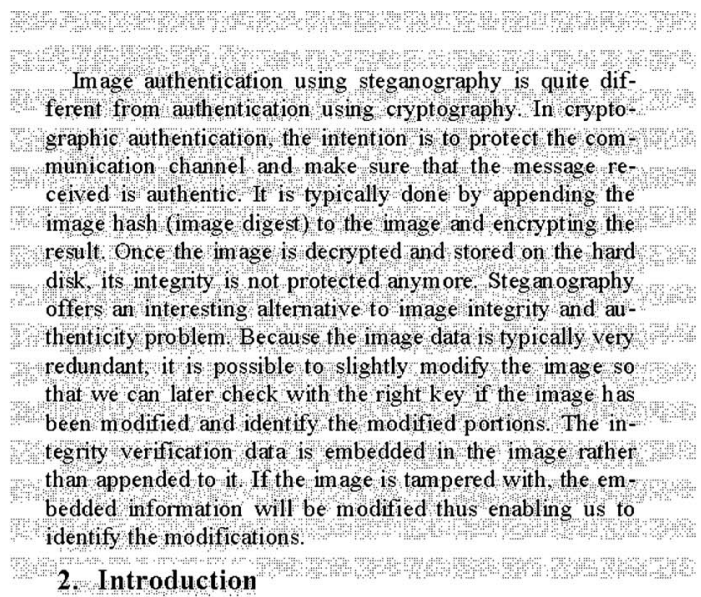

(a) necessary to minimize the visua distortion in the marked images, because minor modification to the pixels can be perceptible since the pixels are either black or white. In this paper, a new perceptual model is proposed for binary images that is useful for data hiding applications. In our model, the distortion that occurs after flipping a pixel is estimated on the novel curvatureweighted distance difference (CWDD) measure between two contour segments: Through subjective tests the perceptual measure is validated and highly correlated with human perception.

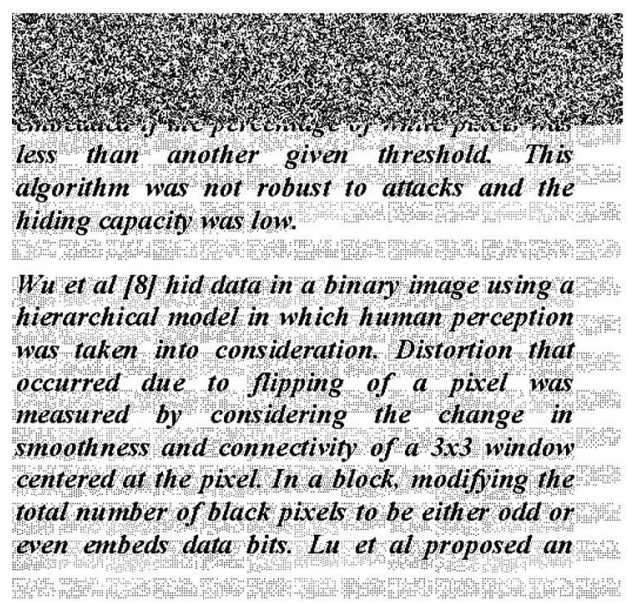

(c) (b)

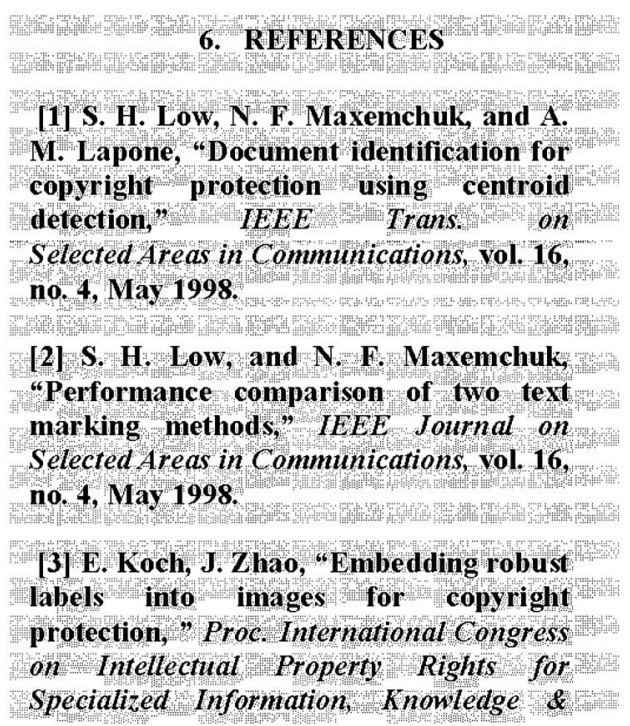

(d)

Fig. 23 (a) to (d) The attacked images.

correction coding should be dependent on the number of characters and text document image size. The adaptive choice of code dimension could enhance the restoration capability of the proposed method. However, it is difficult to convey such an adaptive choice of code dimension to the detector. One possibility is to embed the code dimensions in a robust manner so that it can be extracted during detection. However, this does not guarantee the correct extraction of code dimension in all cases of tampering.

The original and the watermarked images after pixelwise embedding of $m$ in each block are shown in Fig. 13. It is observed that although background noise is present in the watermarked image, the text can be read and understood by the user. Without any tampering, all blocks in the watermarked images are verified. After verification, the original image can be restored at the blind detector. In Fig. 14, it is shown that most blocks in the image have high redundancy for embedding $m$. Due to insufficient redundancy in some blocks, a total of 31 error bits are corrected during ECC decoding, and the original text sequence is extracted. Figure 15 illustrates the number of error bits in each 63-bit segment of the extracted sequence $E_{s}^{d}$ without any attacks. The number of error bits in each segment is found to be lower than the error correction capability. While the error correction capability in each segment is 10 bits, a maximum of 2 error bits are found. However, the error bits introduced due to insufficient redundancy reduces the restoration capability (in the ideal case of sufficient redundancy) against attacks.

We perform multiple modifications such as text deletion, insertion, substitution and block swapping in the watermarked image: (1) Only the word information in the last line is deleted; (2) first three blocks are swapped with the 


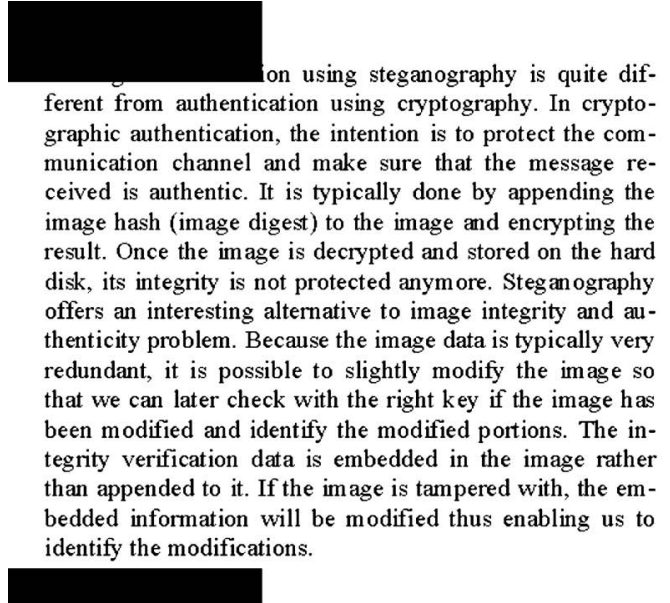

(a)

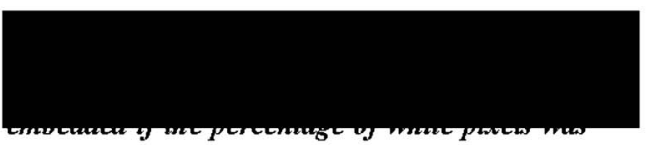

less than another given threshold. This algorithm was not robust to attacks and the hiding capacity was low.

Wu et al [8] hid data in a binary image using a hierarchical model in which human perception was taken into consideration. Distortion that occurred due to flipping of a pixel was measured by considering the change in smoothness and connectivity of a $3 \times 3$ window centered at the pixel. In a block, modifying the total number of black pixels to be either odd or even embeds data bits. Lu et al proposed an

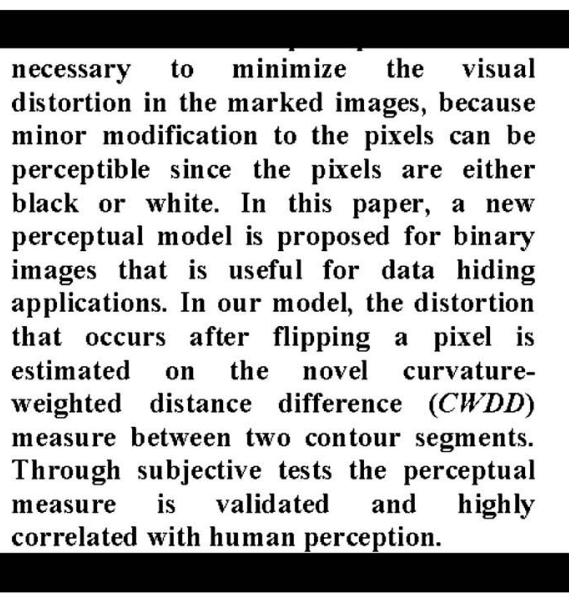

(b)

\section{REFERENCES}

[1] S. H. Low, N. F. Maxemchuk, and A. M. Lapone, "Document identification for copyright protection using centroid detection," IEEE Trans. on

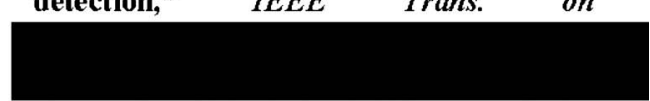

[2] S. H. Low, and N. F. Maxemchuk, "Performance comparison of two text marking methods," IEEE Journal on Selected Areas in Communications, vol. 16, no. 4, May 1998.

[3] E. Koch, J. Zhao, "Embedding robust labels into images for copyright protection," Proc. International Congress on Intellectual Property Rights for Specialized Information, Knowledge \&

Fig. 24 (a) to (d) The images showing the authentic reconstructed blocks; tampered blocks are shown in the dark regions.

last three blocks; (3) the word theory is inserted in the last line; and (4) the word for in line 5 is substituted by the word to. The resulting attacked image is shown in Fig. 16. Blind detection is performed on the attacked image to restore the original text sequence after tamper localization. In Fig. 17, the authentic reconstructed blocks are shown after the watermark erasing process. The detector correctly localizes 13 tampered blocks shown as dark regions. A total of 753 error bits are corrected during ECC decoding, and the original text sequence is extracted. The number of error bits in each 63-bit segment of the extracted sequence $E_{s}^{d}$ is shown in Fig. 18. Since the number of the error bits in each segment does not exceed 10, correct extraction of original text sequence is performed after ECC decoding.

To show the effectiveness of the proposed method, four original images are used in Figs. 19(a)-19(d). The size of the images is $480 \times 560,480 \times 480,512 \times 520$, and 608 $\times 520$ pixels, respectively. The corresponding watermarked images are shown in Fig. 20. The redundancy of different blocks in the original images is shown in Fig. 21. Without any tampering, all blocks in the watermarked images are verified. Fig. 22 illustrates the number of error bits in each 63-bit segment of the extracted sequence $E_{s}^{d}$ from the watermarked images. Due to insufficient redundancy, a total of $56,0,22$, and 8 error bits are corrected during ECC decoding, respectively.

We perform different attacks in the watermarked images, and the attacked images are shown in Figs. 23(a)-23(d). The attacks are described as follows: in the first image, the section title 2. Introduction is moved from the beginning to the end portion. In the second image, the first and last 12 blocks are modified to white pixels. In the third image, the first 39 blocks are modified to uniformly distributed random noise. In the fourth image, the last two sentences of 


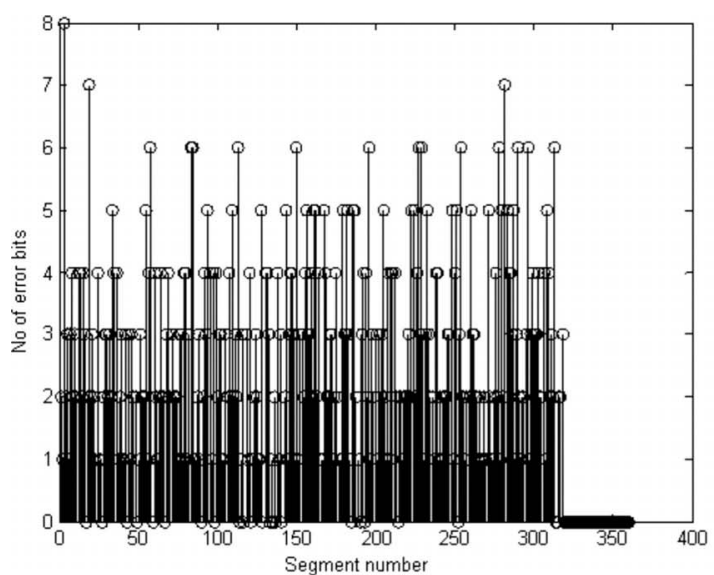

(a)

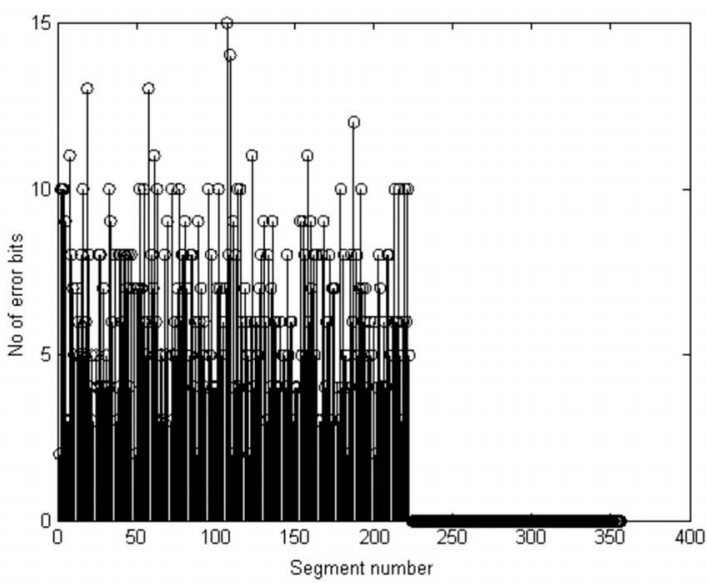

(c)

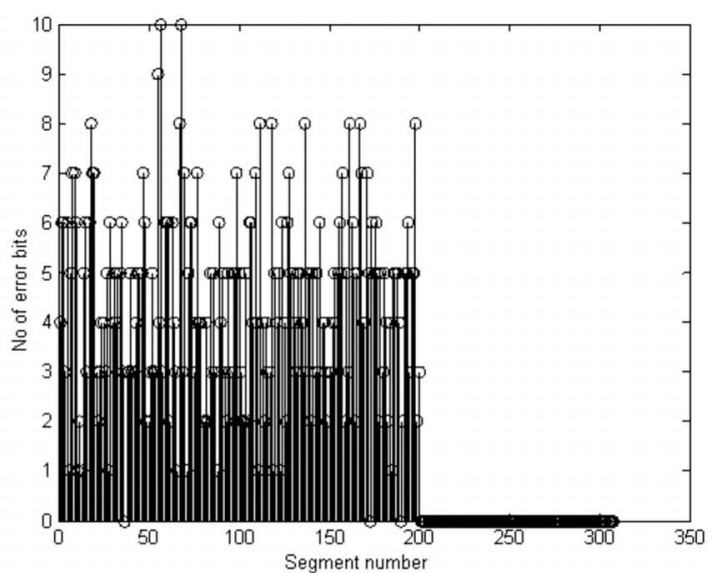

(b)

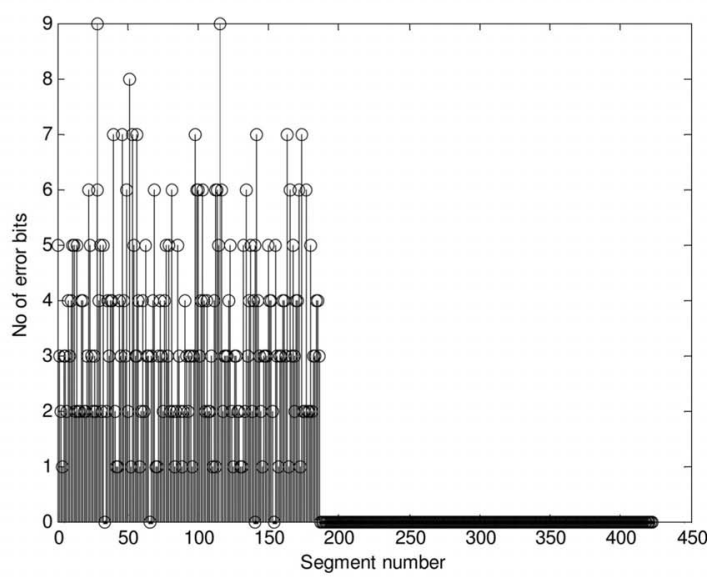

(d)

Fig. 25 (a) to (d) the number of error bits in each 63-bit segment of the bit sequence $E_{s}^{d}$ extracted from the attacked images.

reference [1] are replaced with corresponding sentences of reference [2]. Blind detection is performed on the attacked images to restore the original text sequence after tamper localization. In Fig. 24, the authentic reconstructed blocks are shown after the watermark erasing process for all cases. The detector correctly localizes tampered blocks shown as dark regions. The number of tampered blocks in the attacked images is found to be $15,24,39$, and 26 , respectively. The number of error bits in each 63-bit segment of the extracted sequence $E_{s}^{d}$ is shown in Fig. 25. A total of $832,863,1443$, and 636 error bits are corrected during ECC decoding, respectively. Except in the case of third attacked image, the original text sequence is extracted correctly in all other cases.

Restoration of the original text sequence is not possible during detection if the number of error bits exceeds 10 in any 63-bit segment of the extracted sequence $E_{s}^{d}$. To study restoration failure, we perform an attack by randomly selecting a set of blocks in the watermarked image using a key and the blocks are tampered. Inverse permutation is performed on the extracted sequence $P_{s}^{d}$ to generate $E_{s}^{d}$. The number of error bits in each 63-bit segment of $E_{s}^{d}$ is com- puted. The number of blocks selected for tampering is increased sequentially till the restoration failure occurs, i.e., the total number of error bits exceeds by 10 in any 63-bit segment of $E_{s}^{d}$. The threshold value for an attack (i.e., the number of error bits and error blocks that can be corrected) is found just before reaching the restoration failure. A total of 200 attacks are performed by using various keys in random selection of the blocks. The mean threshold value of the number of error bits and error blocks is found to be 777.95 and 13.92 respectively. In Fig. 26, the histograms of the number of error bits and error blocks are given.

We determine the probability of restoration failure for different values of error bits analytically. The error bits are induced in the extracted sequence $P_{s}^{d}$ after various tampering. The error bits are randomly distributed in $E_{s}^{d}$ due to the permutation procedure. In Ref. 27 , a relevant random permutation analysis is presented to address the uneven embedding capacity equalization problem in watermarking. We compute the threshold value of the error bits at restoration failure using the analysis. The probability that the number of error bits in an $n$-bit segment of $E_{s}^{\prime}$ being $r$ is given by 


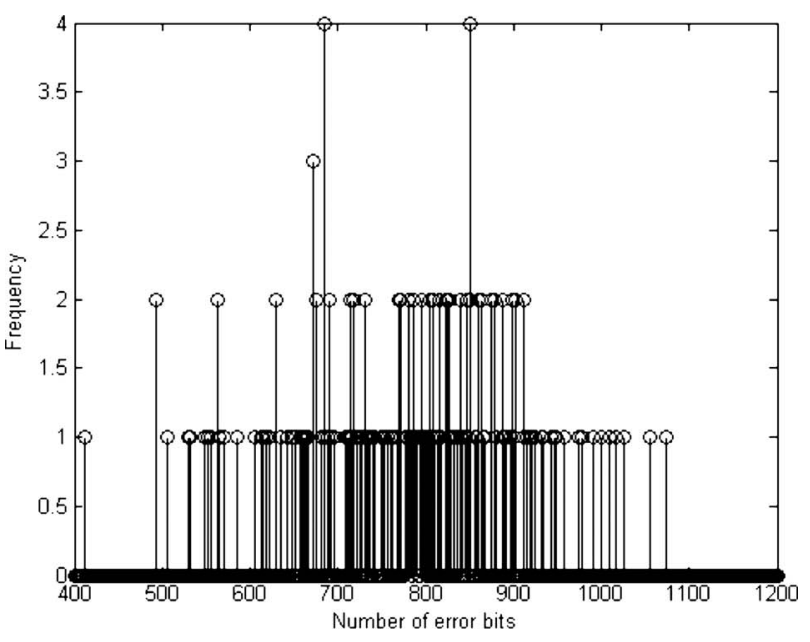

(a)

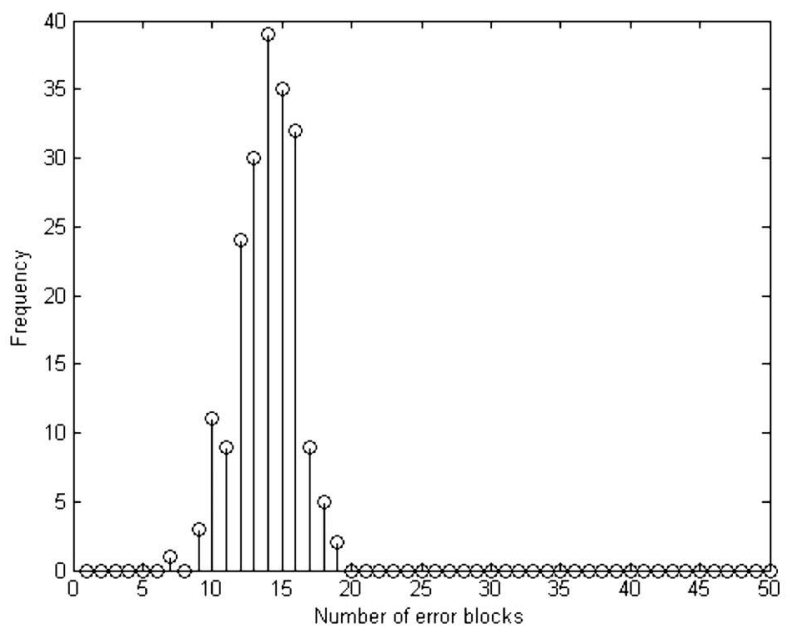

(b)

Fig. 26 Histogram: and (a) The error bits; and (b) the error blocks.

$P_{r}=\frac{\left(\begin{array}{l}n \\ r\end{array}\right)\left(\begin{array}{c}S-n \\ N_{e}-r\end{array}\right)}{\left(\begin{array}{c}S \\ N_{e}\end{array}\right)}$,

where $N_{e}$ is the number of error bits, and $S$ is the length of $E_{s}^{d}$. Restoration failure is possible when the number of error bits in an $n$-bit segment exceeds $t_{s}$. The probability of restoration failure is given by

$P_{T}=\sum_{r=t_{s}+1}^{n} P_{r}$.

In Fig. 27, the plot of $P_{T}$ is shown for different values of $N_{e}$. For $P_{T}=0.01$, the number of error bits that can be corrected is found to be 814 approximately.

The performance of the proposed algorithm can be compared with the previous restoration method for text document images. ${ }^{20}$ In this method, there exists a possibility of false tamper detection and restoration failure. The restora-

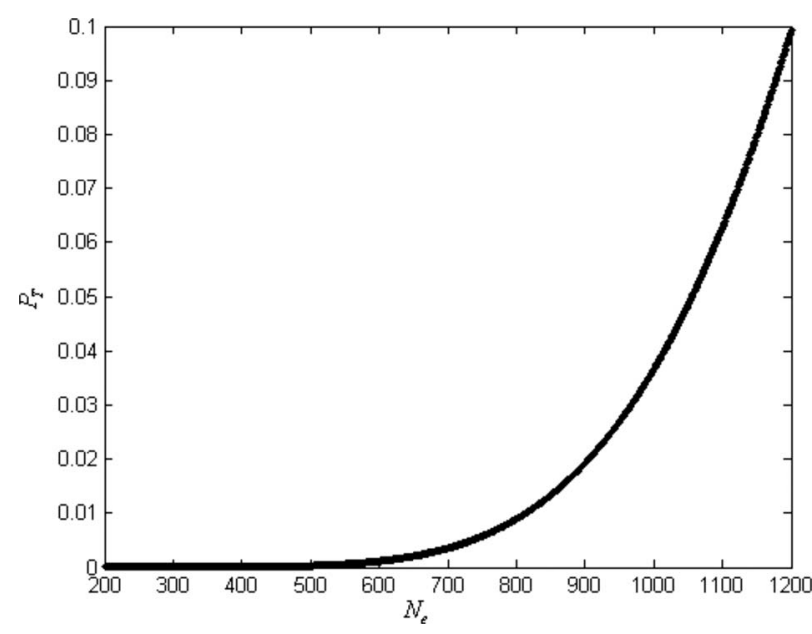

Fig. 27 The plot of $P_{T}$ for different values of $N_{e}$.

tion of a character at any location depends on the watermark which is embedded in another character. The relevant information for restoration is concentrated at certain specific locations rather than being distributed in the whole image. The restoration capability is thus reduced for certain alterations. After multiple alterations such as combined deletion and insertion of characters, restoration would be difficult using this method due to a loss of synchronization. In the proposed method, relevant information is embedded in the whole image in a distributed manner. As such, there is no restoration failure after multiple alterations shown in experimental results. The restoration capability of the proposed method is limited by the number of error bits in the extracted sequence. If the document image is most edited, then ECC coding could not correct the errors beyond a limit.

\section{Conclusion}

In this paper, we proposed a new method for localization and restoration in binary document images using the erasable watermark. The proposed localization method can localize different modifications in the image with high accuracy. To construct the erasable watermark in each block of the original image, an ordered set of insignificant pixels was selected and then compressed using the run-length coding scheme. After the embedding process, the user could interpret the document easily in the presence of noise, incorporating the inherent ability of human vision. After verifying each block, the user could restore the original image for further analysis. The localization accuracy of the proposed method does not suffer from any parity attack and false tamper detection. The new restoration method is particularly useful for restoration of the original text sequence after localization in text document images. In the proposed method, it is possible to restore the original text sequence after multiple alterations such as text deletion, insertion, substitution, and block swapping. Due to the use of fragile watermarks for authentication, the proposed method is particularly useful for binary document images in electronic form. In terms of future work, the proposed restoration method will be extended such that it can be applied to binary document images in general. The erasable water- 
mark could be suitably designed to survive manipulations such as the print and scan, noise, and geometrical deformations.

\section{Acknowledgments}

The authors are very thankful to the reviewers for their useful remarks that helped to improve the paper.

\section{References}

1. I. J. Cox, M. L. Miller, and J. A. Bloom, Digital Watermarking, Morgan Kaufmann Publishers, Inc., San Francisco (2001).

2. S. H. Low, N. F. Maxemchuk, and A. M. Lapone, "Document identification for copyright protection using centroid detection," IEEE Trans. Commun. 46(3), 372-383 (1998).

3. S. H. Low and N. F. Maxemchuk, "Performance comparison of two text marking methods," IEEE J. Sel. Areas Commun. 16(4), 561-572 (1998).

4. J. T. Brassil, S. Low, and N. F. Maxemchuk, "Copyright protection for the electronic distribution of text documents," Proc. IEEE 87(7), 1181-1196 (1999)

5. J. Brassil and L. O'Gorman, "Watermarking document images with bounding box expansion," in Proc. 1st Int. Workshop on Information Hiding, pp. 227-235 (1996).

6. M. Wu and B. Liu, "Data hiding in binary image for authentication and annotation," IEEE Trans. Multimedia 6(4), 528-538 (2004).

7. E. Koch and J. Zhao, "Embedding robust labels into images for copyright protection," in Proc. Int. Congress on Intellectual Property Rights for Specialized Information, Knowledge \& New Technologies (1995).

8. Q. Mei, E. K. Wong, and N. Memon, "Data hiding in binary text documents," in Security and Watermarking of Multimedia Contents III, Proc. SPIE (2001)

9. T. Amamo and D. Misaki, "Feature calibration method for watermarking of document images," in Proc. 5th Int. Conf. Document Analysis and Recognition, pp. 91-94 (1999).

10. A. T. S. Ho, N. B. Puhan, P. Marziliano, A. Makur, and Y. L. Guan, "Perception based binary image watermarking," in Proc. IEEE Int. Symp. Circuits and Systems (ISCAS), vol. 2, pp. 37-40 (2004).

11. N. B. Puhan and A. T. S. Ho, "Secure exact authentication in binary document images," in Proc. IET Intl. Conference on Visual Information Engineering (VIE), pp. 29-34 (2006).

12. Z. Baharav and D. Shaked, "Watermarking of dither half-toned images," in Security and Watermarking of Multimedia Contents, Proc. SPIE 1, 307-313 (1999).

13. M. S. Fu and O. C. Au, "Data hiding watermarking for halftone images," IEEE Trans. Image Process. 11(4), 477-484 (2002).

14. M. Chen, E. Wong, N. Memon, and S. Adams, "Recent developments in Document Image Watermarking and Data Hiding," in Multimedic Systems and Applications IV, Proc. SPIE 4518, 166-176 (2001).

15. N. S. Arjun and A. Negi, "An approach to self-embedding document watermarking based on LZW algorithm," in Proc. IEEE TENCON, pp. 1-4 (2007)

16. C. H. Lin, W. K. Chang, Y, Y. Lin, and L. Y. Cheng "Binary document images authentication by thinning digital patterns," in Proc. Third Int. Conf. on Intelligent Information Hiding and Multimedia Signal Processing (IIHMSP), pp. 485-488 (2007)

17. $\mathrm{S} . \mathrm{Hu}$, "Document image watermarking algorithm based on neighborhood pixel ratio" in Proc. IEEE Int. Conf. Acoustics, Speech, and Signal Processing (ICASSP), vol. 2, pp. 841-844 (2005).

18. H. Y. Kim and R. L. de Queiroz, "Alteration-locating authentication watermarking for binary images," in Proc. Int. Workshop on Digital Watermarking 2004 LNCS-2939 (2004).

19. H. Yang and A. C. Kot, "Binary image authentication with tampering localization by embedding cryptographic signature and block identifier," IEEE Signal Process. Lett. 13(12), 741-744 (2006).

20. A. Makur, "Self-embedding and restoration algorithms for documen watermark," in Proc. IEEE Int. Conf. Acoustics Speech and Signal Processing, vol. 2, pp. 1133-1136 (2005).

21. J. Fridrich, M. Goljan, and M. Du, "Invertible authentication," in Security and Watermarking of Multimedia Contents, spi (2001).

22. K. Sayood, Introduction to Data Compression, Morgan Kauffmann Publishers (2000).
23. P. W. Wong and N. Memon, "Secret and public key image watermarking schemes for image authentication and ownership verification," IEEE Trans. Image Process. 10(10) (2001).

24. R. L. Rivest, "RFC 1321: The MD5 message-digest algorithm," Internet Activities Board (1992).

25. H. Lu, A. C. Kot, and Y. Q. Shi, "Distance-reciprocal distortion measure for binary document images," IEEE Signal Process. Lett. 11(2), 228-231 (2004).

26. R. E. Blahut, Theory and Practice of Error Control Codes, AddisonWesley (1983).

27. M. Wu, Multimedia Data Hiding, PhD Thesis, Princeton University (2001)

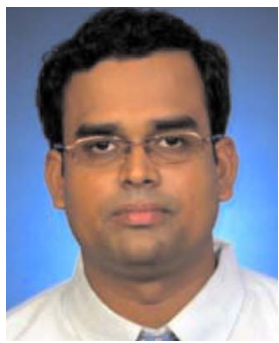

Niladri B. Puhan received his ME degree in signal processing from the Indian Institute of Science (IISc), Bangalore, in 2002 and his PhD from Nanyang Technological University (NTU), Singapore, in 2007. Currently he is a research fellow at NTU. His fields of research interest include digital watermarking, image processing, biometrics and super-resolution image reconstruction.

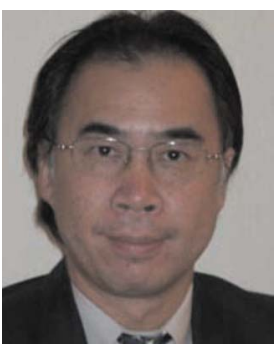

Anthony T. S. Ho joined the Department of Computing, Faculty of Engineering and Physical Sciences, University of Surrey, UK, in 2006. He is a full professor and holds the Personal Chair in Multimedia Security. $\mathrm{He}$ is currently the deputy head of the Department of Computing. Professor Ho cofounded DataMark Technologies (DMT) in 1998. DMT specializes in the research and commercialization of digital watermarking and steganography technologies. He obtained his BSc (Hons) in physical electronics from the University of Northumbria, UK, in 1979, his MSc in applied optics from Imperial College in 1980, and his $\mathrm{PhD}$ in digital image processing from King's College London, University of London, in 1983. Professor $\mathrm{Ho}$ is a fellow of the Institute of Electrical Engineers (FIEE), chartered engineer (CEng), fellow of the Institute of Physics (FInstP), chartered physicist, and a senior member of IEEE. He also serves as a nonexecutive director on the board of DMT. Professor Ho has actively participated in many IEEE and IET committees and conferences as a session chair and technical committee member. He will be the general chair for the 8th International Workshop on Digital Watermarking (IWDW09), which will be held at the University of Surrey in August 2009.

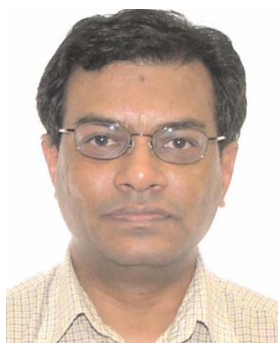

Farook Sattar received his technical license and $\mathrm{PhD}$ degrees in signal and image processing from Lund University, Sweden. His current research interests include watermarking, biomedical signal analysis, blind signal separation, filter banks, wavelets, speech/audio segmentation, image feature extraction, and image enhancement. He had been involved in a number of signal and image processing-related projects sponsored by the Swedish $\mathrm{Na}$ tional Science and Technology Board (NUTEK) and by the Singapore Academic Research Funding (AcRF) Scheme. His research has been published in a number of leading journals and conferences. $\mathrm{He}$ is currently a faculty member in the Information Engineering Division, Nanyang Technological University, Singapore. 